\title{
Robust stabilization by linear output delay feedback
}

\author{
Mark French*
}

\author{
Achim Ilchmann ${ }^{\dagger}$
}

03 June 2008

\author{
Markus Mueller ${ }^{\dagger}$
}

\begin{abstract}
The main result establishes that if a controller $C$ (comprising of a linear feedback of the output and its derivatives) globally stabilizes a (nonlinear) plant $P$, then global stabilization of $P$ can also be achieved by an output feedback controller $C[h]$ where the output derivatives in $C$ are replaced by an Euler approximation with sufficiently small delay $h>0$. This is proved within the conceptual framework of the nonlinear gap metric approach to robust stability. The main result is then applied to finite dimensional linear minimum phase systems with unknown coefficients but known relative degree and known sign of the high frequency gain. Results are also given for systems with non-zero initial conditions.
\end{abstract}

\section{Nomenclature}

$$
\begin{aligned}
& \mathbb{C}_{+}, \mathbb{C}_{-} \quad=\{s \in \mathbb{C} \mid \operatorname{Re} s>0\},\{s \in \mathbb{C} \mid \operatorname{Re} s<0\} \text {, respectively } \\
& |x| \quad=\sqrt{x^{T} x} \text {, the Euclidean norm of } x \in \mathbb{R}^{n} \\
& |A| \quad=\max \left\{|A x|\left|x \in \mathbb{R}^{m},\right| x \mid=1\right\} \text {, the induced matrix norm for } A \in \mathbb{R}^{n \times m} \\
& {\left[a_{1} / a_{2} / \ldots / a_{m}\right]=\left[a_{1}^{T}, a_{2}^{T}, \ldots, a_{m}^{T}\right]^{T} \in \mathbb{R}^{m \times n} \text { for } a_{1}, a_{2}, \ldots, a_{m} \in \mathbb{R}^{1 \times n}} \\
& e_{k}^{(n)} \quad=[0, \ldots, 0,1,0, \ldots, 0]^{T} \text {, the } k \text {-th unit vector in } \mathbb{R}^{n} \text {, for } k, n \in \mathbb{N} \text { and } k \leq n \\
& \operatorname{spec}(A) \quad \text { the spectrum of } A \in \mathbb{R}^{n \times n} \\
& \operatorname{im} A, \operatorname{ker} A \quad \text { the image and kernel of } A \in \mathbb{R}^{n \times m} \\
& \|v\|_{\mathcal{V}} \quad \text { the norm of } v \in \mathcal{V} \text {, for a normed vector space } \mathcal{V} \\
& \operatorname{map}(E \rightarrow F) \quad \text { the set of all maps from the set } E \text { to the set } F \\
& \mathcal{C}^{r}\left(I \rightarrow \mathbb{R}^{\ell}\right) \quad \text { the set of } r \text {-times continuous differentiable functions } y: I \rightarrow \mathbb{R}^{\ell} \text {, where } \\
& r \in \mathbb{N} \cup\{\infty\} \text { and } I \subset \mathbb{R} \text { is an interval } \\
& \mathcal{C}_{\mathrm{pw}}\left(I \rightarrow \mathbb{R}^{\ell}\right) \quad \text { the set of piecewise continuous functions } y: I \rightarrow \mathbb{R}^{\ell}, I \subset \mathbb{R} \text { an interval } \\
& L^{p}\left(I \rightarrow \mathbb{R}^{\ell}\right) \quad \text { the space of } p \text {-integrable functions } y: I \rightarrow \mathbb{R}^{\ell}, 1 \leq p<\infty, I \subset \mathbb{R} \text { an } \\
& \text { interval, with norm }\|y\|_{L^{p}\left(I \rightarrow \mathbb{R}^{\ell}\right)}=\left(\int_{I}|y(t)|^{p} \mathrm{~d} t\right)^{\frac{1}{p}} \\
& L^{\infty}\left(I \rightarrow \mathbb{R}^{\ell}\right) \quad \text { the space of essentially bounded functions } y: I \rightarrow \mathbb{R}^{\ell}, I \subset \mathbb{R} \text { an interval, } \\
& \text { with norm }\|y\|_{L^{\infty}\left(I \rightarrow \mathbb{R}^{\ell}\right)}=\operatorname{ess} \sup _{t \in I}|y(t)| \\
& C L^{p}\left(I \rightarrow \mathbb{R}^{\ell}\right) \quad=\mathcal{C}\left(I \rightarrow \mathbb{R}^{\ell}\right) \cap L^{p}\left(I \rightarrow \mathbb{R}^{\ell}\right), 1 \stackrel{t \in I}{\leq} p \leq \infty, I \subset \mathbb{R} \text { an interval, with norm } \\
& \|y\|_{C L^{p}\left(I \rightarrow \mathbb{R}^{\ell}\right)}=\|y\|_{L^{p}\left(I \rightarrow \mathbb{R}^{\ell}\right)} \\
& C W^{r, p}\left(I \rightarrow \mathbb{R}^{\ell}\right)=\left\{y \in \mathcal{C}^{r}\left(I \rightarrow \mathbb{R}^{\ell}\right) \mid \forall i \in\{0, \ldots, r\}: y^{(i)} \in L^{p}\left(I \rightarrow \mathbb{R}^{\ell}\right)\right\}, r \in \mathbb{N}, 1 \leq p \leq \\
& \infty, I \subset \mathbb{R} \text { an interval, with norm }\|y\|_{C W^{r, p}\left(I \rightarrow \mathbb{R}^{\ell}\right)}=\sum_{i=0}^{r}\left\|y^{(i)}\right\|_{L^{p}\left(I \rightarrow \mathbb{R}^{\ell}\right)}
\end{aligned}
$$

\footnotetext{
${ }^{*}$ School of Electronics and Computer Science, University of Southampton, SO17 1BJ, UK, mcf@ecs.soton.ac.uk

${ }^{\dagger}$ Institut für Mathematik, Technische Universität Ilmenau, Weimarer Straße 25, 98693 Ilmenau, DE, achim.ilchmann, markus.mueller@tu-ilmenau.de
} 


$$
\begin{aligned}
C W_{0}^{r, p}\left(I \rightarrow \mathbb{R}^{\ell}\right)= & \left\{\begin{array}{l|l}
y \in \mathcal{C}^{r}\left(I \rightarrow \mathbb{R}^{\ell}\right) \mid \begin{array}{l}
\forall i \in\{0, \ldots, r\}: y^{(i)} \in L^{p}\left(I \rightarrow \mathbb{R}^{\ell}\right), \\
\text { if } 0 \in I, \text { then } \forall i \in\{0, \ldots, r-1\}: y^{(i)}(0)=0
\end{array}
\end{array}\right\}, \\
r \in \mathbb{N}, 1 \leq p \leq \infty, I \subset \mathbb{R} \text { an interval, with norm }\|y\|_{C W^{r, p}\left(I \rightarrow \mathbb{R}^{\ell}\right)} & \left\{\begin{array}{l}
\forall i \in \mathbb{N}_{0}: y^{(i)} \in L^{p}\left(I \rightarrow \mathbb{R}^{\ell}\right), \\
y \in \mathcal{C}^{\infty}\left(I \rightarrow \mathbb{R}^{\ell}\right) \\
\begin{array}{l}
\sum_{i=0}^{\infty}\left\|y^{(i)}\right\|_{L^{p}\left(I \rightarrow \mathbb{R}^{\ell}\right)}<\infty, \\
\text { if } 0 \in I, \text { then } \forall i \in \mathbb{N}_{0}: y^{(i)}(0)=0
\end{array}
\end{array}\right\}, \\
C W_{0}^{\infty, p}\left(I \rightarrow \mathbb{R}^{\ell}\right)= & 1 \leq p \leq \infty, I \subset \mathbb{R} \text { an interval, with norm }\|y\|_{C W^{\infty, p}\left(I \rightarrow \mathbb{R}^{\ell}\right)}
\end{aligned}
$$

\section{Introduction}

We present conditions under which a feedback controller based on the measured output and its derivatives can be replaced by a feedback controller based on the measured output and numerical derivatives. Derivative feedback occurs frequently in control; for example, PD controllers are of this type as are state feedback of systems of full relative degree and as are suitable partial state feedbacks for systems of non-zero relative degree.

The problem is studied in the setup of the classical feedback configuration shown in Figure 1,

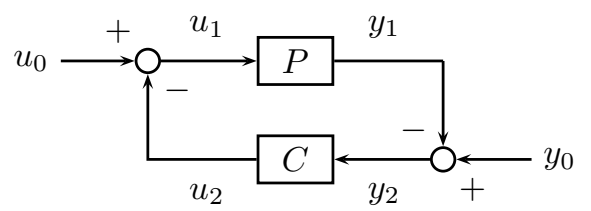

Figure 1: The closed-loop system $[P, C]$

and we are concerned with the concept of gain stability, that is with the existence and size of a finite gain from the external disturbances $\left(u_{0}, y_{0}\right)$ to the internal signals $\left(u_{1}, y_{1}\right)$, that is the quantity

$$
\gamma:=\sup _{\left(u_{0}, y_{0}\right) \in \mathcal{U} \times \mathcal{Y} \backslash\{0\}} \frac{\left\|\left(u_{1}, y_{1}\right)\right\|_{\mathcal{U} \times \mathcal{Y}}}{\left\|\left(u_{0}, y_{0}\right)\right\|_{\mathcal{U} \times \mathcal{Y}}}<\infty,
$$

for some appropriate choices of signal spaces $\mathcal{U}, \mathcal{Y}$. We show that if $P$ is stabilizable $(P$ may be nonlinear) by some derivative feedback controller

$$
C_{k}: y_{2} \mapsto u_{2}=-\sum_{i=0}^{r-1} k_{i+1} y_{2}^{(i)}, \quad k=\left(k_{1}, \ldots, k_{r}\right) \in \mathbb{R}^{1 \times r},
$$

then stabilization can also be achieved by replacing $C_{k}$ by the delay feedback controller $C_{k}^{\text {Euler }}[h]$ for sufficiently small $h>0$, given by

$$
C_{k}^{\text {Euler }}[h]: y_{2} \mapsto u_{2}=-\sum_{i=0}^{r-1} k_{i+1} \Delta_{h}^{i} y_{2},
$$

here $\Delta_{h}^{0} y_{2}=y_{2}$ and $\Delta_{h}^{i} y_{2}$, for $i \geq 1$, denotes the Euler approximation of the $i$ th derivative of $y_{2}$ defined by

$$
\Delta_{h}^{i} y_{2}=\underbrace{\Delta_{h} \circ \cdots \circ \Delta_{h}}_{i \text { times }} y_{2}, \quad \text { where } \quad\left(\Delta_{h} y_{2}\right)(t)=\frac{y_{2}(t)-y_{2}(t-h)}{h} .
$$


The signal spaces for which these results hold depend on structural properties of the plant $P$. For concreteness we consider the case of single input, single output linear plants, which are minimum phase and of relative degree $\rho \geq 1$ and show that the choices $\mathcal{U}=C L^{p}\left(\mathbb{R}_{\geq 0} \rightarrow \mathbb{R}\right)$, $\mathcal{Y}=C W^{k, p}\left(\mathbb{R}_{\geq 0} \rightarrow \mathbb{R}\right)$ are valid, where $k=\rho$ and either $r=\rho-1$ if $p<\infty$ or $r=\rho$ if $p=\infty$. The key motivation for this linear study is to establish the appropriate signal space settings, whereby the degree of regularity required in $\mathcal{Y}$ is determined by the relative degree. In the case of $r=\rho$, a stabilizing high-gain feedback is constructed and an explicit upper bound on the permitted delays is given. The case of $k=\infty$ is also considered. In the linear setting, the results are also extended to incorporate systems with non-zero initial conditions.

The results are established by computing the gap distance between $C_{k}$ and $C_{k}^{\text {Euler }}[h]$ and using variants on nonlinear robust stability theory [7] to deduce the stability of the closed-loop containing the Euler controller from the stability of the derivative feedback controlled closed-loop. In practice, PD controllers or (partial) state feedback are often implemented by such approximations. In the context of nonlinear plants, there are often limited options for the implementation of a (partial) state feedback: nonlinear observers are only available for limited classes of plants. Of course, in practice, a direct implementation of $C_{k}^{\text {Euler }}[h]$ is as problematic as the direct implementation of $C_{k}$ from measurement of $y_{2}$ only: we are simply replacing the problem of calculating the derivative of the measurement with the problem of storing a finite interval of past measurements (so that the delays can be evaluated). However, sampled versions of $C_{k}^{\text {Euler }}[h]$ can also be analysed utilizing the techniques of this paper, and such realisations, which coincide with common engineering practice, give analogous results. A variety of sampled versions of these results will be given in the companion paper [6], which also extends the results for fully nonlinear controllers and to the important case of semi-global stabilization.

Perhaps surprisingly, there are relatively few theoretical results available on closed-loop stability for such delay based controllers. For linear time-invariant systems with relative degree 2 controlled by the delay feedback (1.2), exponential stability of the resulting closed-loop delay differential system was established in [10]. An analogous result for higher relative degree has not been previously established. Stabilization of (nonlinear) systems via delays has been considered by some authors: in [13] the authors give a control strategy with multiple delays that stabilizes a simple system of the form $y^{(n)}=u$. In [12] necessary conditions for multiple delay controllers that stabilize linear systems are shown, but no explicit control strategy is given. In [11] the author considers nonlinear systems with several constraints and gives a control strategy that achieves a bounded output.

The paper is organized as follows. In Section 2 we introduce the background theory and establish a key robust stability result. Section 3 contains the main theorem of the paper which shows that a stabilizing derivative feedback controller may be substituted by a delay feedback controller if the delay is sufficiently small. In Section 4 we consider applications to linear systems to demonstrate structural features of the conditions, giving results establishing both external (gain) stability and internal stability of the closed-loop system.

\section{Background}

The material in this section is based on [7, Sect. II], [4, Sect. 2] and [5, Sect. 2] and contains the gap metric results necessary for proving robustness in Section 3.

\subsection{Terminology}

Let $\mathcal{X}$ be a nonempty set. For $0<\omega \leq \infty$ let $\mathcal{S}_{\omega}$ denote the set of all locally integrable maps in $\operatorname{map}([0, \omega) \rightarrow \mathcal{X})$. For ease of notation define $\mathcal{S}:=\mathcal{S}_{\infty}$. For $0<\tau<\omega \leq \infty$ define a truncation 
operator $T_{\tau}$ and the restriction of maps as follows:

$$
\begin{aligned}
& T_{\tau}: \mathcal{S}_{\omega} \rightarrow \mathcal{S}, \quad v \mapsto T_{\tau} v:=\left(t \mapsto\left\{\begin{array}{ll}
v(t), & t \in[0, \tau) \\
0, & t \in[\tau, \infty)
\end{array}\right),\right. \\
&\left.(\cdot)\right|_{[0, \tau)}: \mathcal{S}_{\omega} \rightarrow \mathcal{S}_{\tau},\left.\quad v \mapsto v\right|_{[0, \tau)}:=(t \mapsto v(t), \quad t \in[0, \tau)) .
\end{aligned}
$$

With $\mathcal{V} \subset \mathcal{S}$ we associate spaces as follows:

$$
\begin{aligned}
\mathcal{V}[0, \tau) & =\left\{v \in \mathcal{S}_{\tau} \mid \exists w \in \mathcal{V} \text { with }\left\|T_{\tau} w\right\|_{\mathcal{V}}<\infty: v=\left.w\right|_{[0, \tau)}\right\}, \text { for } \tau>0 ; \\
\mathcal{V}_{e} & =\left\{v \in \mathcal{S}|\forall \tau>0: v|_{[0, \tau)} \in \mathcal{V}[0, \tau)\right\}, \text { the extended space; } \\
\mathcal{V}_{\omega} & =\left\{v \in \mathcal{S}_{\omega}|\forall \tau \in(0, \omega): v|_{[0, \tau)} \in \mathcal{V}[0, \tau)\right\}, \text { for } 0<\omega \leq \infty ; \\
\mathcal{V}_{a} & =\bigcup_{\omega \in(0, \infty]} \mathcal{V}_{\omega}, \text { the ambient space. }
\end{aligned}
$$

For $L^{p}$ spaces these definitions coincide with the definitions of ambient and extended spaces given in $[4,5,7]$ however note that the definitions in $[4,5,7]$ are not applicable for subspaces of continuously differentiable functions as considered in the present paper, this is due to the fact that $C W^{r, p}\left(\mathbb{R}_{\geq 0} \rightarrow \mathbb{R}\right)$ is not closed under the action of $T_{\tau}, \tau>0$.

If $v, w \in \mathcal{V}_{a}$ with $\left.v\right|_{I}=\left.w\right|_{I}$ on $I=\operatorname{dom}(v) \cap \operatorname{dom}(w)$, then we write $v=w$. For $(u, y) \in \mathcal{V}_{a} \times \mathcal{V}_{a}$, the domains of $u$ and $y$ may be different; we adopt the convention

$$
\operatorname{dom}(u, y):=\operatorname{dom}(u) \cap \operatorname{dom}(y) .
$$

We say $\mathcal{V} \subset \mathcal{S}$ is a signal space if, and only if, it is a normed vector space. For our main results we will consider different types of signal spaces which are specified in (3.2).

\subsection{Well posedness}

A mapping $Q: \mathcal{U}_{a} \rightarrow \mathcal{Y}_{a}$ is said to be causal if, and only if,

$$
\forall x, y \in \mathcal{U}_{a} \forall \tau \in \operatorname{dom}(x) \cap \operatorname{dom}(Q x): \quad\left[\left.x\right|_{[0, \tau)}=\left.\left.y\right|_{[0, \tau)} \Rightarrow(Q x)\right|_{[0, \tau)}=\left.(Q y)\right|_{[0, \tau)}\right] .
$$

Consider $P: \mathcal{U}_{a} \rightarrow \mathcal{Y}_{a}, u_{1} \mapsto y_{1}$, and $C: \mathcal{Y}_{a} \rightarrow \mathcal{U}_{a}, y_{2} \mapsto u_{2}$ being causal mappings representing the plant and the controller, respectively, and satisfying the closed-loop equations:

$$
[P, C]: \quad y_{1}=P u_{1}, \quad u_{2}=C y_{2}, \quad u_{0}=u_{1}+u_{2}, \quad y_{0}=y_{1}+y_{2},
$$

corresponding to the closed-loop shown in Figure 1.

For $w_{0}=\left(u_{0}, y_{0}\right) \in \mathcal{W}:=\mathcal{U} \times \mathcal{Y}$ a pair $\left(w_{1}, w_{2}\right)=\left(\left(u_{1}, y_{1}\right),\left(u_{2}, y_{2}\right)\right) \in \mathcal{W}_{a} \times \mathcal{W}_{a}, \mathcal{W}_{a}:=\mathcal{U}_{a} \times \mathcal{Y}_{a}$, is a solution if, and only if, (2.1) holds on $\operatorname{dom}\left(w_{1}, w_{2}\right)$. The (possibly empty) set of solutions is denoted by

$$
\mathcal{X}_{w_{0}}:=\left\{\left(w_{1}, w_{2}\right) \in \mathcal{W}_{a} \times \mathcal{W}_{a} \mid\left(w_{1}, w_{2}\right) \text { solves }(2.1)\right\}
$$

The closed-loop system $[P, C]$, given by $(2.1)$, is said to have:

- the existence property if, and only if, $\mathcal{X}_{w_{0}} \neq \emptyset$;

- the uniqueness property if, and only if,

$$
\begin{aligned}
& \forall w_{0} \in \mathcal{W}:\left[\left(\hat{w}_{1}, \hat{w}_{2}\right),\left(\tilde{w}_{1}, \tilde{w}_{2}\right) \in \mathcal{X}_{w_{0}} \Longrightarrow\right. \\
& \left.\left(\hat{w}_{1}, \hat{w}_{2}\right)=\left(\tilde{w}_{1}, \tilde{w}_{2}\right) \quad \text { on } \quad \operatorname{dom}\left(\hat{w}_{1}, \hat{w}_{2}\right) \cap \operatorname{dom}\left(\tilde{w}_{1}, \tilde{w}_{2}\right)\right] .
\end{aligned}
$$


Assume that $[P, C]$ has the existence and uniqueness property. For each $w_{0} \in \mathcal{W}$, define $\omega_{w_{0}} \in$ $(0, \infty]$, by the property

$$
\left[0, \omega_{w_{0}}\right):=\cup_{\left(\hat{w}_{1}, \hat{w}_{2}\right) \in \mathcal{X}_{w_{0}}} \operatorname{dom}\left(\hat{w}_{1}, \hat{w}_{2}\right)
$$

and define $\left(w_{1}, w_{2}\right) \in \mathcal{W}_{a} \times \mathcal{W}_{a}$, with $\operatorname{dom}\left(w_{1}, w_{2}\right)=\left[0, \omega_{w_{0}}\right)$, by the property $\left.\left(w_{1}, w_{2}\right)\right|_{[0, t)} \in \mathcal{X}_{w_{0}}$ for all $t \in\left[0, \omega_{w_{0}}\right)$. This construction induces the operator

$$
H_{P, C}: \mathcal{W} \rightarrow \mathcal{W}_{a} \times \mathcal{W}_{a}, \quad w_{0} \mapsto\left(w_{1}, w_{2}\right)
$$

For $\Omega \subset \mathcal{W}$ the closed-loop system $[P, C]$, given by $(2.1)$, is said to be:

- locally well posed on $\Omega$ if, and only if, it has the existence and uniqueness properties and the operator $\left.H_{P, C}\right|_{\Omega}: \Omega \rightarrow \mathcal{W}_{a} \times \mathcal{W}_{a}, w_{0} \mapsto\left(w_{1}, w_{2}\right)$, is causal;

- globally well posed on $\Omega$ if, and only if, it is locally well posed on $\Omega$ and $H_{P, C}(\Omega) \subset \mathcal{W}_{e} \times \mathcal{W}_{e}$;

- regularly well posed if, and only if, it is locally well posed and

$$
\forall w_{0} \in \mathcal{W} \quad\left[\omega_{w_{0}}<\infty \quad \Rightarrow \quad\left\|\left.\left(H_{P, C} w_{0}\right)\right|_{[0, \tau)}\right\|_{\mathcal{W}_{\tau} \times \mathcal{W}_{\tau}} \rightarrow \infty \text { as } \tau \rightarrow \omega_{w_{0}}\right] .
$$

\subsection{Graphs, the nonlinear gap metric and gain stability}

For the plant operator $P: \mathcal{U}_{a} \rightarrow \mathcal{Y}_{a}$ and the controller operator $C: \mathcal{Y}_{a} \rightarrow \mathcal{U}_{a}$ define the graph $\mathcal{G}_{P}$ of the plant and the graph $\mathcal{G}_{C}$ of the controller, respectively, as follows:

$$
\mathcal{G}_{P}:=\left\{\left(\begin{array}{c}
u \\
P u
\end{array}\right) \mid u \in \mathcal{U}, P u \in \mathcal{Y}\right\} \subset \mathcal{W}, \quad \mathcal{G}_{C}:=\left\{\left(\begin{array}{c}
C y \\
y
\end{array}\right) \mid C y \in \mathcal{U}, y \in \mathcal{Y}\right\} \subset \mathcal{W} .
$$

Note that $\mathcal{G}_{P}$ and $\mathcal{G}_{C}$ are, strictly speaking, not subsets of $\mathcal{W}$; however, abusing the notation we identify $\mathcal{G}_{P} \ni\left(\begin{array}{c}u \\ P u\end{array}\right)=(u, P u) \in \mathcal{W}$ and $\mathcal{G}_{C} \ni\left(\begin{array}{c}C y \\ y\end{array}\right)=(C y, y) \in \mathcal{W}$. An operator $P: \mathcal{U}_{a} \rightarrow \mathcal{Y}_{a}$ is said to be causally extendible [8] (or stabilizable in [4]) if, and only if,

$$
\forall \tau>0 \forall w_{1}=\left(u_{1}, y_{1}\right) \in \mathcal{W}_{a} \text { with } T_{\tau} y_{1}=T_{\tau} P u_{1} \exists w_{1}^{*} \in \mathcal{G}_{P}: T_{\tau} w_{1}=T_{\tau} w_{1}^{*} .
$$

Given normed signal spaces $\mathcal{X}$ and $\mathcal{V}$ and $\Omega \subset \mathcal{X}$, a causal operator $Q: \mathcal{X} \rightarrow \mathcal{V}_{a}$ is said to be gain stable on $\Omega$ if, and only if,

$Q(\Omega) \subset \mathcal{V}, \quad Q(0)=0, \quad\left\|\left.Q\right|_{\Omega}\right\|_{\mathcal{X}, \mathcal{V}}:=\sup \left\{\frac{\left\|\left.(Q x)\right|_{[0, \tau)}\right\| \mathcal{V}_{\tau}}{\left\|\left.x\right|_{[0, \tau)} \mid\right\|_{\mathcal{X}_{\tau}}}|x \in \Omega, \tau>0, x|_{[0, \tau)} \neq 0\right\}<\infty$

Given normed signal spaces $\mathcal{U}, \mathcal{Y}$ and $\mathcal{W}:=\mathcal{U} \times \mathcal{Y}$ and causal operators $P: \mathcal{U}_{a} \rightarrow \mathcal{Y}_{a}, C: \mathcal{Y}_{a} \rightarrow$ $\mathcal{U}_{a}$ we make the following definitions. The closed-loop system $[P, C]$ given by $(2.1)$ with the associated operator $H_{P, C}: \mathcal{W} \rightarrow \mathcal{W}_{a} \times \mathcal{W}_{a}$ is said to be $\mathcal{W}$-stable if, and only if, it is globally well posed and $H_{P, C}(\mathcal{W}) \subset \mathcal{W} \times \mathcal{W}$. It is said to be $\mathcal{W}$-gain stable if and only if it is $\mathcal{W}$-stable and $H_{P, C}$ is gain stable on $\mathcal{W}$.

Next, associate with the closed-loop system $[P, C]$ given by $(2.1)$ the following two parallel projection operators:

$$
\Pi_{P / / C}: \mathcal{W} \rightarrow \mathcal{W}_{a}, w_{0} \mapsto w_{1} \quad \text { and } \quad \Pi_{C / / P}: \mathcal{W} \rightarrow \mathcal{W}_{a}, w_{0} \mapsto w_{2} .
$$

Note that gain stability of either $\Pi_{P / / C}$ and $\Pi_{C / / P}$ implies gain stability of the closed-loop system $[P, C]$, and that $\left\|\Pi_{P / / C}\right\|_{\mathcal{W}, \mathcal{W}},\left\|\Pi_{C / / P}\right\|_{\mathcal{W}, \mathcal{W}} \geq 1$ since $\Pi_{P / / C}=\Pi_{P / / C}^{2}, \Pi_{C / / P}=\Pi_{C / / P}^{2}$. 
For $P_{1}, P_{2} \in \Gamma(\mathcal{U}, \mathcal{Y}):=\left\{P: \mathcal{U}_{a} \rightarrow \mathcal{Y}_{a} \mid P\right.$ is causal $\}$, define the directed gap by

$$
\vec{\delta}: \Gamma(\mathcal{U}, \mathcal{Y}) \times \Gamma(\mathcal{U}, \mathcal{Y}) \rightarrow[0, \infty], \quad \vec{\delta}\left(P_{1}, P_{2}\right):=\inf _{\Phi \in \mathcal{O}_{P_{1}, P_{2}}} \sup _{x \in \mathcal{G}_{P_{1}} \backslash\{0\}}\left(\frac{\left\|\left.(\Phi-I)\right|_{\mathcal{G}_{P_{1}}}(x)\right\|_{\mathcal{W}}}{\|x\|_{\mathcal{W}}}\right)
$$

where $\mathcal{O}_{P_{1}, P_{2}}$ is the (possibly empty) set

$$
\mathcal{O}_{P_{1}, P_{2}}:=\left\{\Phi: \mathcal{G}_{P_{1}} \rightarrow \mathcal{G}_{P_{2}} \mid \Phi \text { is causal, surjective and } \Phi(0)=0\right\} .
$$

Here we adopt the convention that $\vec{\delta}\left(P_{1}, P_{2}\right):=\infty$ if $\mathcal{O}_{P_{1}, P_{2}}=\emptyset$. The nonlinear gap is defined as

$$
\delta: \Gamma(\mathcal{U}, \mathcal{Y}) \times \Gamma(\mathcal{U}, \mathcal{Y}) \rightarrow[0, \infty], \quad\left(P_{1}, P_{2}\right) \mapsto \delta\left(P_{1}, P_{2}\right):=\max \left\{\vec{\delta}\left(P_{1}, P_{2}\right), \vec{\delta}\left(P_{2}, P_{1}\right)\right\}
$$

\subsection{Robust Stability}

We now prove the robust stability theorem on which the main result in this paper is based. This result is based on [7, Th. 1], but extends the scope of that result in several directions. Firstly, the result is established in the language of ambient signal spaces to handle finite escape times (cf. [7, Th. 8]). More importantly, the implicit requirement in [7] of well posedness of $\left[P_{1}, C\right]$ is extended to include the often weaker requirement of regular well posedness. This eases the application of the result in general, as global well posedness is non-trivial to verify a priori, and regular well posedness is often easier to establish (for $p=\infty$ regular well posedness follows from standard results on the finite escape time properties of differential equations).

Note that we state this theorem in a form where stability of $\left[P_{1}, C\right]$ is inferred from $[P, C]$, however, in the sequel we will apply this theorem in the setting whereby stability of $\left[P, C_{1}\right]$ is to be inferred from $[P, C]$. Such applications of the theorem follow from a trivial interchange of $P$ and $C$ and $\mathcal{U}, \mathcal{Y}$; we elect to present the theorem in the context of $P, P_{1}$ to follow the convention of the literature and since, in contrast to this paper, most applications of such robust stability results concern uncertainty in the plant $P$.

Theorem 2.1 Let $\mathcal{U}, \mathcal{Y}$ be signal spaces and $\mathcal{W}=\mathcal{U} \times \mathcal{Y}$. Consider $P: \mathcal{U}_{a} \rightarrow \mathcal{Y}_{a}, P_{1}: \mathcal{U}_{a} \rightarrow \mathcal{Y}_{a}$ and $C: \mathcal{Y}_{a} \rightarrow \mathcal{U}_{a}$ with $P(0)=0, C(0)=0$. Suppose $[P, C]$ is gain stable on $\mathcal{W}, P_{1}$ is causally extendible and $\left[P_{1}, C\right]$ is either a) globally or b) regularly well posed. If

$$
\vec{\delta}\left(P, P_{1}\right)<\left\|\Pi_{P / / C}\right\|_{\mathcal{W}, \mathcal{W}}^{-1}
$$

then the closed-loop system $\left[P_{1}, C\right]$ is gain stable on $\mathcal{W}$ with

$$
\left\|\Pi_{P_{1} / / C}\right\|_{\mathcal{W}, \mathcal{W}} \leq\left\|\Pi_{P / / C}\right\|_{\mathcal{W}, \mathcal{W}} \frac{1+\vec{\delta}\left(P, P_{1}\right)}{1-\left\|\Pi_{P / / C}\right\|_{\mathcal{W}, \mathcal{W}} \vec{\delta}\left(P, P_{1}\right)}
$$

Proof. Since $\left\|\Pi_{P / / C}\right\|_{\mathcal{W}, \mathcal{W}} \geq 1$, it follows that $\vec{\delta}\left(P, P_{1}\right)<\infty$ and hence there exists a causal surjective mapping $\Phi: \mathcal{G}_{P} \rightarrow \mathcal{G}_{P_{1}}$ such that

$$
\gamma:=\left\|(\Phi-I) \Pi_{P / / C}\right\| \leq\|(\Phi-I)\| \cdot\left\|\Pi_{P / / C}\right\|<1 .
$$

Let $w \in \mathcal{W}$ and let $\left[0, \omega_{w}\right)$ be the maximal interval of existence for $H_{P_{1}, C}(w)$. Let $0<\tau<\omega_{w}$. Consider the equation

$$
\begin{aligned}
\left.w\right|_{[0, \tau)} & =\left.\left(\left(I+(\Phi-I) \Pi_{P / / C}\right)(x)\right)\right|_{[0, \tau)} \\
& =\left.\left(\left(\Pi_{C / / P}+\Phi \Pi_{P / / C}\right)(x)\right)\right|_{[0, \tau)} .
\end{aligned}
$$


By either well posedness assumption a) or b), we know that $\left[P_{1}, C\right]$ is locally well posed, and hence satisfies the existence and uniqueness properties on $[0, \tau)$. Hence there exists $w_{1}=$ $\left(u_{1}, y_{1}\right), w_{2}=\left(u_{2}, y_{2}\right) \in \mathcal{W}_{\omega_{w}}$ such that $y_{1}=P_{1} u_{1}, u_{2}=C y_{2}$ and $\left.w\right|_{[0, \tau)}=\left.w_{1}\right|_{[0, \tau)}+\left.w_{2}\right|_{[0, \tau)}$. Since $P_{1}$ is stabilizable, there exists $w_{1}^{\prime \prime} \in \mathcal{G}_{P_{1}}$, such that $\left.w_{1}^{\prime \prime}\right|_{[0, \tau)}=\left.w_{1}\right|_{[0, \tau)}$. By definition of $\mathcal{W}_{\omega_{w}}$ we have $\left.w_{2}\right|_{[0, \tau)} \in \mathcal{W}[0, \tau)$ and hence there exists $w_{2}^{\prime} \in \mathcal{W}$ such that $\left.w_{2}^{\prime}\right|_{[0, \tau)}=\left.w_{2}\right|_{[0, \tau)}$. Since $\Phi$ is surjective it follows that there exists $w_{1}^{\prime} \in \mathcal{G}_{P}$ such that $\Phi\left(w_{1}^{\prime}\right)=w_{1}^{\prime \prime}$ and hence $\left.\left(\Phi\left(w_{1}^{\prime}\right)\right)\right|_{[0, \tau)}=\left.w_{1}^{\prime \prime}\right|_{[0, \tau)}=\left.w_{1}\right|_{[0, \tau)}$. It can now be seen that $x=w_{1}^{\prime}+w_{2}^{\prime} \in \mathcal{W}$ satisfies $\left.x\right|_{[0, \tau)}=\left.\left(w_{1}^{\prime}+w_{2}\right)\right|_{[0, \tau)}$ and $x$ is a solution of (2.7).

Since $\Phi, \Pi_{P_{1} / / C}, \Pi_{P / / C}, \Pi_{C / / P}$ are causal, it follows from equation (2.7) that

$$
\left.\left(\Pi_{P_{1} / / C}(w)\right)\right|_{[0, \tau)}=\left.\left(\Pi_{P_{1} / / C}\left(\Pi_{C / / P} x+\Phi \Pi_{P / / C}(x)\right)\right)\right|_{[0, \tau)}=\left.\left(\Phi \Pi_{P / / C}(x)\right)\right|_{[0, \tau)} .
$$

It follows from (2.6) that $\left\|\left.x\right|_{[0, \tau)}\right\| \leq \frac{1}{1-\gamma}\left\|\left.w\right|_{[0, \tau)}\right\|$, hence, in view of (2.3), (2.5) and (2.8),

$$
\begin{aligned}
\left\|\left.\Pi_{P_{1} / / C}(w)\right|_{[0, \tau)}\right\| & =\left\|\left.\Phi \Pi_{P / / C}(x)\right|_{[0, \tau)}\right\| \\
& \leq\left\|\left.\Pi_{P / / C}(x)\right|_{[0, \tau)}\right\|+\left\|\left.(\Phi-I) \Pi_{P / / C}(x)\right|_{[0, \tau)}\right\| \\
& \leq\left\|\Pi_{P / / C}\right\|_{\mathcal{W}, \mathcal{W}} \frac{1+\vec{\delta}\left(P, P_{1}\right)}{1-\left\|\Pi_{P / / C}\right\|_{\mathcal{W}, \mathcal{W}} \vec{\delta}\left(P, P_{1}\right)}\left\|\left.w\right|_{[0, \tau)}\right\| .
\end{aligned}
$$

If $\left[P_{1}, C\right]$ is globally well posed, $\omega_{w}=\infty$, so inequality (2.9) holds for all $\tau>0$, and the proof is complete.

Suppose $\left[P_{1}, C\right]$ is regularly well posed. Since we have shown $\left(\left.\Pi_{P_{1} / / C}(w)\right|_{[0, \tau)} \in \mathcal{W}[0, \tau)\right.$ is uniformly bounded for all $\tau \in\left(0, \omega_{w}\right)$ and since $\left[P_{1}, C\right]$ is regularly well posed, it follows that $\omega_{w}=\infty$ so inequality (2.9) holds for all $\tau>0$. This completes the proof.

\section{Robust stabilization by delay feedback}

Our main result will establish conditions under which a derivative feedback controller (1.1) may be replaced by Euler approximation (1.2). We first formally define, for $h>0$, the Euler approximation

$$
\begin{aligned}
\Delta_{h}: \operatorname{map}\left(\mathbb{R}_{\geq 0} \rightarrow \mathbb{R}\right) & \rightarrow \operatorname{map}\left(\mathbb{R}_{\geq 0} \rightarrow \mathbb{R}\right), \\
(t \mapsto y(t)) & \mapsto\left(t \mapsto \frac{y(t)-y(t-h)}{h}\right), \quad \text { where } y(s)=0 \text { if } s<0,
\end{aligned}
$$

of the derivative of $y$ and, for higher derivatives $y^{(i)}, i \in \mathbb{N}$,

$$
\begin{aligned}
\Delta_{h}^{i}: \operatorname{map}\left(\mathbb{R}_{\geq 0} \rightarrow \mathbb{R}\right) & \rightarrow \operatorname{map}\left(\mathbb{R}_{\geq 0} \rightarrow \mathbb{R}\right), \\
y & \mapsto \Delta_{h}^{i}(y):= \begin{cases}\Delta_{h}^{i-1}\left(\Delta_{h}(y)\right) & \text { if } i \geq 2 \\
\Delta_{h}(y) & \text { if } i=1 \\
y & \text { if } i=0 .\end{cases}
\end{aligned}
$$


Our results will hold in the following signal space settings $(A)-(C)$ :

$$
\begin{array}{rll}
\text { (A) } \mathcal{W} & =\mathcal{U} \times \mathcal{Y}=C L^{\infty}\left(\mathbb{R}_{\geq 0} \rightarrow \mathbb{R}\right) & \times C W^{r, \infty}\left(\mathbb{R}_{\geq 0} \rightarrow \mathbb{R}\right), \\
\mathcal{W}_{0}=\mathcal{U}_{0} \times \mathcal{Y}_{0}=C L^{\infty}\left(\mathbb{R}_{\geq 0} \rightarrow \mathbb{R}\right) & \times C W_{0}^{r, \infty}\left(\mathbb{R}_{\geq 0} \rightarrow \mathbb{R}\right), r \in \mathbb{N}, p=\infty \\
\text { (B) } \mathcal{W}=\mathcal{U} \times \mathcal{Y}=C L^{p}\left(\mathbb{R}_{\geq 0} \rightarrow \mathbb{R}\right) & \times C W^{r, p}\left(\mathbb{R}_{\geq 0} \rightarrow \mathbb{R}\right), \\
\mathcal{W}_{0}=\mathcal{U}_{0} \times \mathcal{Y}_{0}=C L^{p}\left(\mathbb{R}_{\geq 0} \rightarrow \mathbb{R}\right) & \times C W_{0}^{r, p}\left(\mathbb{R}_{\geq 0} \rightarrow \mathbb{R}\right), \quad r \in \mathbb{N}, p \in[1, \infty), \\
\text { (C) } \mathcal{W}=\mathcal{U} \times \mathcal{Y}=C W^{\infty, p}\left(\mathbb{R}_{\geq 0} \rightarrow \mathbb{R}\right) \times C W^{\infty, p}\left(\mathbb{R}_{\geq 0} \rightarrow \mathbb{R}\right), \\
\mathcal{W}_{0}=\mathcal{U}_{0} \times \mathcal{Y}_{0}=C W_{0}^{\infty, p}\left(\mathbb{R}_{\geq 0} \rightarrow \mathbb{R}\right) \times C W_{0}^{\infty, p}\left(\mathbb{R}_{\geq 0} \rightarrow \mathbb{R}\right), p \in[1, \infty) .
\end{array}
$$

The spaces $\mathcal{W}_{0}$ will be utilized for results whereby the initial conditions of the system are zero, whilst the spaces $\mathcal{W}$ are utilized in the general setting with non-zero initial conditions. The spaces of type (A) and (B) are standard, the need for spaces with constrained derivatives arises from the setting whereby derivative based controllers are being considered. In Section 4 we will motivate the spaces of type $(\mathrm{C})$, which allows for more general controllers (we will not require $k_{r}=0$ for controller $C_{k}$ given by (1.1) as for signal spaces of type (B), see below) at the price of greater regularity constraints on the disturbances.

We are now in a position to state the main result of this section, namely that if $C_{k}$ gain stabilizes $P$ it follows that $C_{k}^{\text {Euler }}[h]$ is also a gain stabilizing controller of $P$ for sufficiently small $h>0$. The idea behind the proof is to show that the gap $\vec{\delta}\left(C_{k}, C_{k}^{\text {Euler }}[h]\right)$ is small if $h>0$ is small and hence deduce the result from Theorem 2.1.

Theorem 3.1 Let $1 \leq p \leq \infty, r \in \mathbb{N}$ and consider signal spaces $\mathcal{U}_{0}, \mathcal{Y}_{0}$ and $\mathcal{W}_{0}$ of type (A), (B) or (C) in (3.2). Suppose that there exists $k=\left(k_{1}, \ldots, k_{r}\right) \in \mathbb{R}^{1 \times r} \backslash\{0\}$, with $k_{r}=0$ for case (B), such that controller $C_{k}: \mathcal{Y}_{0 a} \rightarrow \mathcal{U}_{0 a}$ given by (1.1) applied to a causal plant $P: \mathcal{U}_{0 a} \rightarrow \mathcal{Y}_{0 a}$ with $P(0)=0$ yields a closed-loop system $\left[P, C_{k}\right]$ which is gain stable on $\mathcal{W}_{0}:=\mathcal{U}_{0} \times \mathcal{Y}_{0}$. Suppose $h^{*}>0$ satisfies

$h^{*} \leq\left(\gamma \sum_{i=1}^{r-1}\left|k_{i+1}\right| \cdot i \eta_{p}\left(h^{*}, i\right)\right)^{-1}$ where, for $h>0, \eta_{p}(h, i):= \begin{cases}1, & \text { in case (A) } \\ 2^{\frac{1}{p}}(1+i h p)^{\frac{1}{p}} & \text { in case (B) } \\ 2^{\frac{1}{p}}(1+i h p) & \text { in case (C) }\end{cases}$

and $1 \leq \gamma:=\left\|\Pi_{C_{k} / / P}\right\|_{\mathcal{W}_{0}, \mathcal{W}_{0}}<\infty$. Let $h \in\left(0, h^{*}\right)$ and suppose that $\left[P, C_{k}^{\text {Euler }}[h]\right]$ is either globally or regularly well posed, where the controller $C_{k}^{\text {Euler }}[h]: \mathcal{Y}_{0 a} \rightarrow \mathcal{U}_{0 a}$ is given by (1.2). Then the closed-loop system $\left[P, C_{k}^{\text {Euler }}[h]\right]$ is gain stable on $\mathcal{W}_{0}$ with

$$
\left\|\Pi_{C_{k}^{\text {Euler }[h] / / P}}\right\|_{\mathcal{W}_{0}, \mathcal{W}_{0}} \leq\left\|\Pi_{C_{k} / / P}\right\|_{\mathcal{W}_{0}, \mathcal{W}_{0}} \frac{1+h \sum_{i=1}^{r-1}\left|k_{i+1}\right| i \eta_{p}(h, i)}{1-\left\|\Pi_{C_{k} / / P}\right\|_{\mathcal{W}_{0}, \mathcal{W}_{0}} h \sum_{i=1}^{r-1}\left|k_{i+1}\right| i \eta_{p}(h, i)} .
$$

In all three signal space settings (A), (B) and (C) the condition (3.3) on $h^{*}$ can always be met for sufficiently small $h^{*}>0$, e.g. by taking $h^{*}=\left(\gamma \sum_{i=1}^{r-1}\left|k_{i+1}\right| \cdot i\right)^{-1}$ in case (A) and by taking $h^{*}=\min \left\{\frac{1}{r p},\left(2^{\frac{1+p}{p}} \gamma \sum_{i=1}^{r-1}\left|k_{i+1}\right| \cdot i\right)^{-1}\right\}$ in cases (B) and (C). Before giving the proof of this result, we establish the following key bound which will be required in the proof of Theorem 3.1 for the signal space choices (B) and (C). 
Proposition 3.2 For $y \in \mathcal{C}\left(\mathbb{R}_{\geq 0} \rightarrow \mathbb{R}\right)$ and $\varrho>0$, define the function

$$
M_{\varrho}[y]: \mathbb{R}_{\geq 0} \rightarrow \mathbb{R}, \quad t \mapsto \max _{\tau \in[t-\varrho, t]}|y(\tau)|, \quad \text { where } y(s)=0 \text { if } s<0 .
$$

Then, for every $y \in C W_{0}^{1, p}\left(\mathbb{R}_{\geq 0} \rightarrow \mathbb{R}\right)$ and $1 \leq p<\infty$,

$$
\forall T>0:\left\|M_{\varrho}[y]\right\|_{L^{p}([0, T) \rightarrow \mathbb{R})}^{p} \leq 2\|y\|_{L^{p}\left(\mathbb{R}_{\geq 0} \rightarrow \mathbb{R}\right)}^{p-1}\left(\|y\|_{L^{p}\left(\mathbb{R}_{\geq 0} \rightarrow \mathbb{R}\right)}+\varrho p\|\dot{y}\|_{L^{p}\left(\mathbb{R}_{\geq 0} \rightarrow \mathbb{R}\right)}\right) .
$$

Proof. Let $y \in C W_{0}^{1, p}\left(\mathbb{R}_{\geq 0} \rightarrow \mathbb{R}\right), 1 \leq p<\infty, T>0, \varepsilon>0, i \in \mathbb{N}_{0}$. By the density of $\left.C_{0}^{2}([i, i+1]) \rightarrow \mathbb{R}\right)$ in $C_{0}([i, i+1] \rightarrow \mathbb{R})$ it follows from [15, Th. 4.12] applied successively on the intervals $[i, i+1]$ that there exists a (piecewise cubic) function $G_{i}:[i, i+1] \rightarrow \mathbb{R}$ such that $G_{i}$ is nowhere locally constant, $G_{i} \in C^{2}([i, i+1], \mathbb{R}), G_{i}(i)=y(i), \dot{G}_{i}(i)=\dot{y}(i), G_{i}(i+1)=y(i+1)$, $\dot{G}_{i}(i+1)=\dot{y}(i+1)$, and

$$
\left|G_{i}(t)-y(t)\right| \leq \frac{\varepsilon}{2^{i+1}} \leq \varepsilon e^{-t}, \quad\left|\dot{G}_{i}(t)-\dot{y}(t)\right| \leq \frac{\varepsilon}{2^{i+1}} \leq \varepsilon e^{-t}, \quad t \in[i, i+1] .
$$

We now define $G \in C^{1}\left(\mathbb{R}_{\geq 0}, \mathbb{R}\right)$ by $\left.G\right|_{[i, i+1]}=\left.G_{i}\right|_{[i, i+1]}, i \in \mathbb{N}_{0}$. Suppose, for the time being,

$$
\left\|M_{\varrho}[G]\right\|_{L^{p}([0, T) \rightarrow \mathbb{R})}^{p} \leq 2\|G\|_{L^{p}\left(\mathbb{R}_{\geq 0} \rightarrow \mathbb{R}\right)}^{p-1}\left(\|G\|_{L^{p}\left(\mathbb{R}_{\geq 0} \rightarrow \mathbb{R}\right)}+\varrho p\|\dot{G}\|_{L^{p}\left(\mathbb{R}_{\geq 0} \rightarrow \mathbb{R}\right)}\right) .
$$

Then in view of

$$
M_{\varrho}[y](t)=M_{\varrho}[y+G-G](t) \leq M_{\varrho}[G](t)+M_{\varrho}[y-G](t) \leq M_{\varrho}[G](t)+M_{\varrho}\left[\epsilon e^{-\cdot}\right](t),
$$

and $M_{\varrho}\left[\epsilon e^{-\cdot}\right](t)=\varepsilon e^{-t}$ for $t \geq 0$, it follows that

$$
\left\|M_{\varrho}[y]\right\|_{L^{p}([0, T) \rightarrow \mathbb{R})}^{p} \leq\|G\|_{L^{p}\left(\mathbb{R}_{\geq 0} \rightarrow \mathbb{R}\right)}^{p-1}\left(\|G\|_{L^{p}\left(\mathbb{R}_{\geq 0} \rightarrow \mathbb{R}\right)}+\varrho p\|\dot{G}\|_{L^{p}\left(\mathbb{R}_{\geq 0} \rightarrow \mathbb{R}\right)}\right)+\frac{\varepsilon^{p}}{p} .
$$

Since

$$
\begin{aligned}
& \|G\| \leq\|G-y\|_{L^{p}\left(\mathbb{R}_{\geq 0} \rightarrow \mathbb{R}\right)}+\|y\|_{L^{p}\left(\mathbb{R}_{\geq 0} \rightarrow \mathbb{R}\right)} \leq c \varepsilon+\|y\|_{L^{p}\left(\mathbb{R}_{\geq 0} \rightarrow \mathbb{R}\right)}, \\
& \|\dot{G}\| \leq\|\dot{G}-\dot{y}\|_{L^{p}\left(\mathbb{R}_{\geq 0} \rightarrow \mathbb{R}\right)}+\|\dot{y}\|_{L^{p}\left(\mathbb{R}_{\geq 0} \rightarrow \mathbb{R}\right)} \leq c \varepsilon+\|\dot{y}\|_{L^{p}\left(\mathbb{R}_{\geq 0} \rightarrow \mathbb{R}\right)},
\end{aligned}
$$

where $c:=(1 / p)^{1 / p}$, it follows that

$$
\begin{aligned}
& \left\|M_{\varrho}[y](t)\right\|_{L^{p}([0, T) \rightarrow \mathbb{R})}^{p} \\
& \quad \leq\left(c \varepsilon+\|y\|_{L^{p}\left(\mathbb{R}_{\geq 0} \rightarrow \mathbb{R}\right)}\right)^{p-1}\left(c \varepsilon+\|y\|_{L^{p}\left(\mathbb{R}_{\geq 0} \rightarrow \mathbb{R}\right)}+\varrho p\left(c \varepsilon+\|\dot{y}\|_{L^{p}\left(\mathbb{R}_{\geq 0} \rightarrow \mathbb{R}\right)}\right)\right)+\frac{\varepsilon^{p}}{p} .
\end{aligned}
$$

As this holds for all $\varepsilon>0$, inequality (3.6) follows as required.

It remains to show (3.7). Let

$$
\mathcal{R}(G):=\left\{t \in \mathbb{R}_{\geq 0}|| G(t) \mid \text { is a local maximum of }|G|\right\} .
$$

Since $|G|$ is piecewise polynomial, $G \not \equiv 0, \mathcal{R}(G)$ is non-empty and has a finite or countable number of elements. To every point $t \in \mathcal{R}(G)$ we define (adopting the convention that $\inf \emptyset=\infty$ )

$$
\begin{aligned}
t^{M} & :=\inf \{\tau>t|| G(\tau) \mid \text { is a local minimum of }|G|\}, \\
t^{R} & :=\min \{t+\varrho, \inf \{\tau \in \mathcal{R}(G) \mid \tau>t\}\} .
\end{aligned}
$$




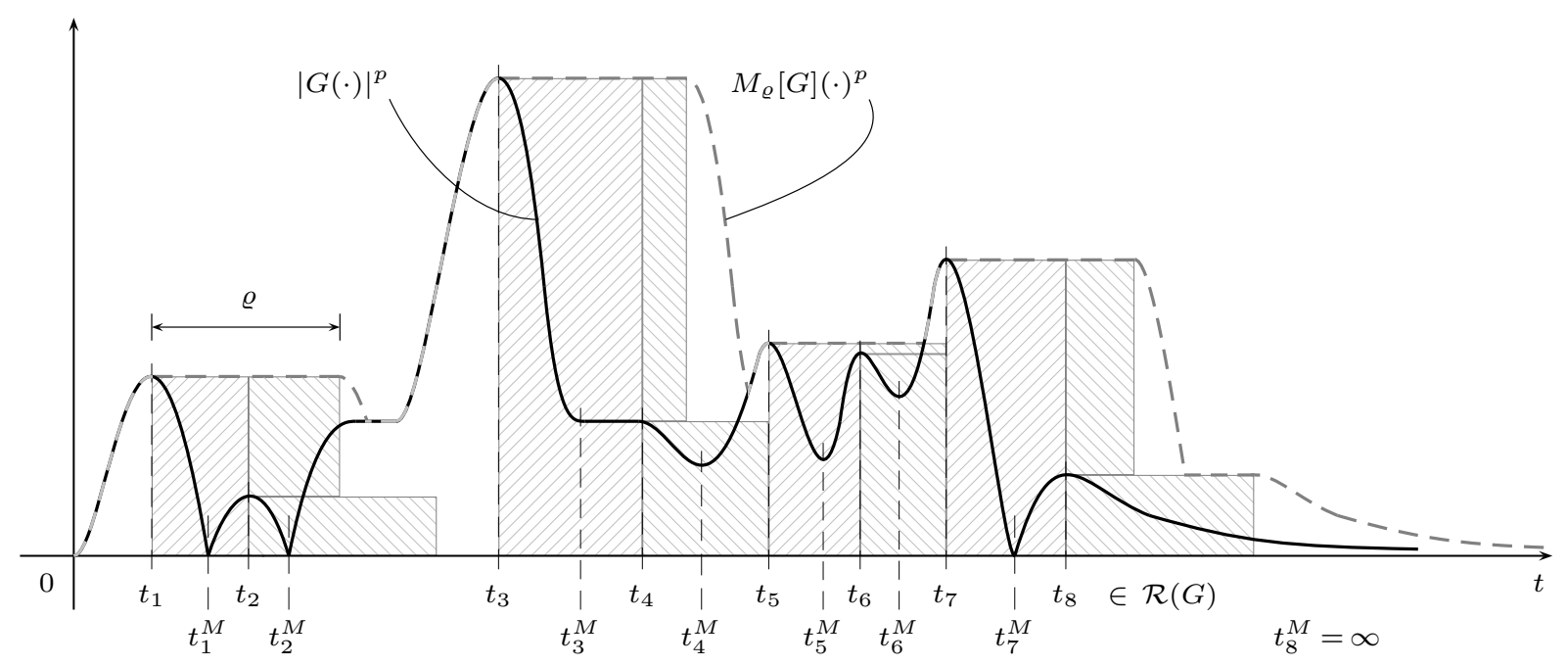

Figure 2: Example function $|G|^{p}$ and $M_{\varrho}[G]^{p}$, here: $t_{1}^{R}=t_{2}, t_{2}^{R}=t_{2}+\varrho, t_{3}^{R}=t_{4}, t_{4}^{R}=t_{5}$, $t_{5}^{R}=t_{6}, t_{6}^{R}=t_{7}, t_{7}^{R}=t_{8}$ and $t_{8}^{R}=t_{8}+\varrho$.

We estimate the $L^{p}$-norm of $M_{\varrho}[G]$ by the $L^{p}$-norm of $G$ and the sum of parts of the areas of the hatched boxes, see Figure 2. By the definition of $M_{\varrho}[G]$ we have

$$
\begin{aligned}
\left\|M_{\varrho}[G]\right\|_{L^{p}([0, T) \rightarrow \mathbb{R})}^{p} & \int_{0}^{T}\left(\max _{\tau \in[t-\varrho, t]}|G(\tau)|\right)^{p} \mathrm{~d} t \\
\leq & \int_{0}^{\infty}|G(t)|^{p} \mathrm{~d} t+\sum_{t \in \mathcal{R}(G)}( \\
& \left(\left[t^{R}-t\right]|G(t)|^{p}\right. \\
& \left.\quad+\left[t+\varrho-t^{R}\right] \cdot \max \left\{0,|G(t)|^{p}-\left|G\left(t^{R}\right)\right|^{p}\right\}\right),
\end{aligned}
$$

where $\left(\left[t^{R}-t\right]|G(t)|^{p}\right)$ is the area of the hatched box of height $|G(t)|^{p}$ between the local maximum $t$ and either the following local maximum $t^{R}$ on the right or the point $t+\varrho$. Furthermore, $\left[t+\varrho-t^{R}\right] \cdot \max \left\{0,|G(t)|^{p}-\left|G\left(t^{R}\right)\right|^{p}\right\}$ is the area of the box which remains by subtracting a box with the height $\left|G\left(t^{R}\right)\right|^{p}$ of the following maximum value $t^{R}$ from a box with height $|G(t)|^{p}$ and length $t+\varrho-t^{R}$. Since $\left|G\left(t^{R}\right)\right| \geq\left|G\left(t^{M}\right)\right|$ and since $\left(t, t^{M}\right) \cap\left(s, s^{M}\right)=\emptyset$ for all $t, s \in \mathcal{R}(G)$, we have:

$$
\sum_{t \in \mathcal{R}(G)}\left[t^{R}-t\right]\left|G\left(t^{M}\right)\right|^{p} \leq \sum_{t \in \mathcal{R}(G)} \int_{t}^{t^{R}}|G(t)|^{p} \mathrm{~d} t \leq \int_{0}^{\infty}|G(t)|^{p} \mathrm{~d} t
$$

and hence

$$
\begin{aligned}
&\left\|M_{\varrho}[G]\right\|_{L^{p}([0, T) \rightarrow \mathbb{R})}^{p} \leq \int_{0}^{\infty}|G(t)|^{p} \mathrm{~d} t+\sum_{t \in \mathcal{R}(G)}\left(\left[t^{R}-t\right]\left(|G(t)|^{p}-\left|G\left(t^{M}\right)\right|^{p}\right)+\left[t^{R}-t\right]\left|G\left(t^{M}\right)\right|^{p}\right. \\
&\left.+\left[t+\varrho-t^{R}\right] \cdot \max \left\{0,|G(t)|^{p}-\left|G\left(t^{R}\right)\right|^{p}\right\}\right) \\
& \leq \int_{0}^{\infty}|G(t)|^{p} \mathrm{~d} t+\sum_{t \in \mathcal{R}(G)}\left(\left[t^{R}-t\right]\left(|G(t)|^{p}-\left|G\left(t^{M}\right)\right|^{p}\right)+\left[t^{R}-t\right]\left|G\left(t^{M}\right)\right|^{p}\right. \\
&\left.+\left[t+\varrho-t^{R}\right]\left(|G(t)|^{p}-\left|G\left(t^{M}\right)\right|^{p}\right)\right) \\
& \leq 2 \int_{0}^{\infty}|G(t)|^{p} \mathrm{~d} t+\varrho \sum_{t \in \mathcal{R}(G)}\left(|G(t)|^{p}-\left|G\left(t^{M}\right)\right|^{p}\right) .
\end{aligned}
$$


Since $\left.G\right|_{\left(t, t^{M}\right)}$ is either strictly positive or negative, $|G|$ is continuously differentiable on $\left(t, t^{M}\right)$, and partial integration yields

$$
\sum_{t \in \mathcal{R}(G)}\left(|G(t)|^{p}-\left|G\left(t^{M}\right)\right|^{p}\right) \leq \sum_{t \in \mathcal{R}(G)} \int_{t}^{t^{M}} p|G(t)|^{p-1}|\dot{G}(t)| \mathrm{d} t \leq p\left\|G^{p-1} \dot{G}\right\|_{L^{1}\left(\mathbb{R}_{\geq 0} \rightarrow \mathbb{R}\right)},
$$

where the second inequality above follows from $\left(t, t^{M}\right) \cap\left(s, s^{M}\right)=\emptyset$ for all $t, s \in \mathcal{R}(G)$. Let $1<q<\infty$ satisfy $\frac{1}{p}+\frac{1}{q}=1$, then by Hölder's inequality

$$
\left\|G^{p-1} \dot{G}\right\|_{L^{1}\left(\mathbb{R}_{\geq 0} \rightarrow \mathbb{R}\right)} \leq\left\|G^{p-1}\right\|_{L^{q}\left(\mathbb{R}_{\geq 0} \rightarrow \mathbb{R}\right)}\|\dot{G}\|_{L^{p}\left(\mathbb{R}_{\geq 0} \rightarrow \mathbb{R}\right)}=\|G\|_{L^{p}\left(\mathbb{R}_{\geq 0} \rightarrow \mathbb{R}\right)}^{p-1}\|\dot{G}\|_{L^{p}\left(\mathbb{R}_{\geq 0} \rightarrow \mathbb{R}\right)}
$$

Finally, inequalities (3.8), (3.9) and (3.10) give the claimed inequality (3.7).

We are now in a position to prove Theorem 3.1.

Proof of Theorem 3.1. Let $1 \leq p \leq \infty, r \in \mathbb{N}$, signal spaces $\mathcal{U}_{0}, \mathcal{Y}_{0}$ and $\mathcal{W}_{0}$ of type (A), (B) or (C) given by $(3.2)$, and $k=\left(k_{1}, \ldots, k_{r}\right) \in \mathbb{R}^{1 \times r}, k_{r}=0$ in case (B). We claim that if $h \in\left(0, h^{*}\right)$, then

$$
\vec{\delta}\left(C_{k}, C_{k}^{\text {Euler }}[h]\right) \leq h \sum_{i=1}^{r-1}\left|k_{i+1}\right| \cdot i \eta_{p}(h, i),
$$

and hence,

$$
\vec{\delta}\left(C_{k}, C_{k}^{\text {Euler }}[h]\right) \stackrel{(3.11)}{\leq} h \sum_{i=0}^{r-1}\left|k_{i+1}\right| \cdot i \eta_{p}(h, i) \stackrel{(3.3)}{<} \gamma^{-1}=\left\|\Pi_{C_{k} / / P}\right\|_{\mathcal{W}_{0}, \mathcal{W}_{0}}^{-1} .
$$

By assumption, $P, C$ are causal, $C(0)=P(0)=0,\left[P, C_{k}\right]$ is gain stable on $\mathcal{W}_{0}$ and $\left[P, C_{k}^{\text {Euler }}[h]\right]$ is either globally or regularly well posed. Finally, since $C_{k}^{\text {Euler }}[h]\left(\mathcal{Y}_{0}\right) \subset \mathcal{U}_{0}$ it follows that $C_{k}^{\text {Euler }}[h]$ is causally extendible. Applying Theorem 2.1 with the roles of $P$ and $C$ interchanged we see that (3.4) is a consequence of inequality (2.4) and inequality (3.11).

It remains to show (3.11).

Step 1: The graphs of $C_{k}$ and $C_{k}^{\text {Euler }}[h]$ are given by

$$
\begin{aligned}
\mathcal{G}_{C_{k}} & =\left\{\left(\begin{array}{c}
-\sum_{i=0}^{r-1} k_{i+1} y^{(i)} \\
y
\end{array}\right) \mid-\sum_{i=0}^{r-1} k_{i+1} y^{(i)} \in \mathcal{U}, y \in \mathcal{Y}\right\} \subset \mathcal{U} \times \mathcal{Y}, \\
\mathcal{G}_{C_{k}^{\text {Euler }[h]}} & =\left\{\left(\begin{array}{c}
-\sum_{i=0}^{r-1} k_{i+1} \Delta_{h}^{i}(y) \\
y
\end{array}\right) \mid-\sum_{i=0}^{r-1} k_{i+1} \Delta_{h}^{i}(y) \in \mathcal{U}, y \in \mathcal{Y}\right\} \subset \mathcal{U} \times \mathcal{Y} .
\end{aligned}
$$

Consider the surjective map

$$
\Phi_{h}: \mathcal{G}_{C_{k}} \rightarrow \mathcal{G}_{C_{k}^{\text {Euler }[h]}}, \quad\left(\begin{array}{c}
-\sum_{i=0}^{r-1} k_{i+1} y^{(i)} \\
y
\end{array}\right) \mapsto\left(\begin{array}{c}
-\sum_{i=0}^{r-1} k_{i+1} \Delta_{h}^{i}(y) \\
y
\end{array}\right) .
$$

Since $\left\|\left(-\sum_{i=0}^{r-1} k_{i+1} y^{(i)}, y\right)^{T}\right\|_{\mathcal{U}_{0} \times \mathcal{Y}_{0}} \geq\|y\|_{\mathcal{Y}_{0}}$ and

$$
\begin{aligned}
\left\|\left(\sum_{i=0}^{r-1} k_{i+1} \Delta_{h}^{i}(y), y\right)^{T}-\left(\sum_{i=0}^{r-1} k_{i+1} y^{(i)}, y\right)^{T}\right\|_{\mathcal{W}_{0}} & =\left\|\sum_{i=1}^{r-1} k_{i+1}\left(\Delta_{h}^{i}(y)-y^{(i)}\right)\right\|_{\mathcal{U}_{0}} \\
& \leq \sum_{i=1}^{r-1}\left|k_{i+1}\right|\left\|\Delta_{h}^{i}(y)-y^{(i)}\right\|_{\mathcal{U}_{0}},
\end{aligned}
$$


it follows that

$$
\vec{\delta}\left(C_{k}, C_{k}^{\text {Euler }}[h]\right) \leq\left\|\Phi_{h}-I\right\|_{\mathcal{W}_{0}, \mathcal{W}_{0}} \leq \sup _{y \in \mathcal{Y}_{0} \backslash\{0\}} \frac{\sum_{i=1}^{r-1}\left|k_{i+1}\right|\left\|\Delta_{h}^{i}(y)-y^{(i)}\right\|_{\mathcal{U}_{0}}}{\|y\|_{\mathcal{Y}_{0}}} .
$$

Note that (3.13) holds for all signal spaces $\mathcal{U}_{0}$ and $\mathcal{Y}_{0}$ considered in $(\mathrm{A}),(\mathrm{B})$ and $(\mathrm{C})$.

Step 2: Recall, that, for $y \in \mathcal{Y}_{0}$, the definition of $\mathcal{Y}_{0}$ gives $y^{(i)}(0)=0$, for all $i \in\{0, \ldots, r-1\}$, in case of $(\mathrm{A})$ and $(\mathrm{B})$, and that $y^{(i)}(0)=0$, for all $i \in \mathbb{N}_{0}$ in case of $(\mathrm{C})$. Also recall that by definition of $\Delta_{h}^{i}$ we have $\Delta_{h}^{i}(y)(t)=0$ for $t<i h$. To simplify notation, without loss of generality, define $y(t)=0$ for $t<0$.

Let $y \in \mathcal{Y}_{0}$ and fix $i \in\{1, \ldots, r-1\}$. By $i+1$ applications of the Mean Value Theorem there exist, for $j \in\{1, \ldots, i\}$, functions $\xi_{j}^{i}:[0, \infty) \rightarrow \mathbb{R}$ with $\xi_{j}^{i}(t) \in(0, j h]$ and $\xi_{i+1}^{i, 0}:[0, \infty) \rightarrow \mathbb{R}$ with $\xi_{i+1}^{i, 0}(t) \in(0, i h]$ such that, for all $t \geq 0$,

$$
\begin{aligned}
\left|\Delta_{h}^{i}(y)(t)-y^{(i)}(t)\right| & =\left|\Delta_{h}^{i-1}\left(\frac{1}{h}(y(\cdot)-y(\cdot-h))\right)(t)-y^{(i)}(t)\right| \\
& =\left|\Delta_{h}^{i-1} y^{(1)}\left(t-\xi_{1}^{i}\right)-y^{(i)}(t)\right| \\
& \vdots \\
& =\left|\frac{1}{h}\left(y^{(i-1)}\left(t-\xi_{i-1}^{i}\right)-\left(y^{(i-1)}\right)\left(t-\xi_{i-1}^{i}-h\right)\right)-y^{(i)}(t)\right| \\
& =\left|y^{(i)}\left(t-\xi_{i}^{i}\right)-y^{(i)}(t)\right| \\
& \leq i h\left|y^{(i+1)}\left(t-\xi_{i+1}^{i, 0}(t)\right)\right| .
\end{aligned}
$$

Furthermore, in case of $(\mathrm{C})$ there exist, for all $\mu \in \mathbb{N}$, functions $\xi_{i+1}^{i, \mu}:[0, \infty) \rightarrow \mathbb{R}$ with $\xi_{i+1}^{i, \mu}(t) \in$ $(0, i h]$ such that, for all $t \geq 0$,

$$
\left|\Delta_{h}^{i}\left(y^{(\mu)}\right)(t)-y^{(\mu+i)}(t)\right| \leq i h\left|y^{(\mu+i+1)}\left(t-\xi_{i+1}^{i, \mu}(t)\right)\right| .
$$

Hence: in case (A) for $p=\infty, \mu=0$; in case (B) for $p \in[1, \infty), \mu=0$; and in case (C) for $p \in[1, \infty), \mu \in \mathbb{N}_{0}$; the following inequality holds

$$
\begin{aligned}
\left\|\Delta_{h}^{i}\left(y^{(\mu)}\right)-y^{(\mu+i)}\right\|_{L^{p}\left(\mathbb{R}_{\geq 0} \rightarrow \mathbb{R}\right)} & \leq i h\left\|y^{(\mu+i+1)}\left(\cdot-\xi_{i+1}^{i, \mu}(\cdot)\right)\right\|_{L^{p}\left(\mathbb{R}_{\geq 0} \rightarrow \mathbb{R}\right)} \\
& \leq i h\left\|M_{i h}\left[y^{(\mu+i+1)}\right](\cdot)\right\|_{L^{p}\left(\mathbb{R}_{\geq 0} \rightarrow \mathbb{R}\right)} .
\end{aligned}
$$

Step 3: We show inequality (3.11) in case (A), i.e. for $\mathcal{U}_{0}=C L^{\infty}\left(\mathbb{R}_{\geq 0} \rightarrow \mathbb{R}\right)$ and $\mathcal{Y}_{0}=$ $C W_{0}^{r, \infty}\left(\mathbb{R}_{\geq 0} \rightarrow \mathbb{R}\right)$. Let $y \in \mathcal{Y}_{0}$. Observe that $\left\|y^{(i+1)}\right\|_{\mathcal{U}_{0}} \leq\|y\|_{\mathcal{Y}_{0}}$ for all $i \in\{1, \ldots, r-1\}$. Thus it follows from inequalities (3.13), (3.14) that

$$
\vec{\delta}\left(C_{k}, C_{k}^{\text {Euler }}[h]\right) \leq \sup _{y \in \mathcal{Y}_{0} \backslash\{0\}} \frac{\sum_{i=1}^{r-1}\left|k_{i+1}\right| i h\left\|y^{(i+1)}\right\|_{\mathcal{U}_{0}}}{\|y\|_{\mathcal{Y}_{0}}} \leq h \sum_{i=1}^{r-1}\left|k_{i+1}\right| i .
$$

This completes the proof in case of $(\mathrm{A})$. 
Step 4: We show (3.11) in case (B) with $k_{r}=0$, that is for $p \in[1, \infty)$ we let $\mathcal{U}_{0}=C L^{p}\left(\mathbb{R}_{\geq 0} \rightarrow \mathbb{R}\right)$ and $\mathcal{Y}_{0}=C W_{0}^{r, p}\left(\mathbb{R}_{\geq 0} \rightarrow \mathbb{R}\right)$. Let $y \in \mathcal{Y}_{0}, i \in\{1, \ldots, r-2\}$. Since $k=\left(k_{1}, \ldots, k_{r-1}, 0\right) \in \mathbb{R}^{1 \times r}$ and $y^{(i+1)} \in C W_{0}^{1, p}\left(\mathbb{R}_{\geq 0} \rightarrow \mathbb{R}\right)$, it follows from (3.14) and Proposition 3.2 that

$$
\begin{aligned}
\left\|\Delta_{h}^{i}(y)-y^{(i)}\right\|_{\mathcal{U}_{0}} & \leq i h\left\|M_{i h}\left[y^{(i+1)}\right](\cdot)\right\|_{\mathcal{U}_{0}} \\
& \leq i h\left(2\left\|y^{(i+1)}\right\|_{\mathcal{U}_{0}}^{p-1}\left(\left\|y^{(i+1)}\right\|_{\mathcal{U}_{0}}+i h p\left\|y^{(i+2)}\right\|_{\mathcal{U}_{0}}\right)\right)^{\frac{1}{p}} \\
& \leq 2^{\frac{1}{p}} i h(1+i h p)^{\frac{1}{p}}\|y\|_{\mathcal{Y}_{0}}
\end{aligned}
$$

Then by (3.13) and (3.15)

$$
\vec{\delta}\left(C_{k}, C_{k}^{\text {Euler }}[h]\right) \leq 2^{\frac{1}{p}} h \sum_{i=0}^{r-2}\left|k_{i+1}\right| \cdot i(1+i h p)^{\frac{1}{p}} \stackrel{k_{r}=0}{=} 2^{\frac{1}{p}} h \sum_{i=0}^{r-1}\left|k_{i+1}\right| \cdot i(1+i h p)^{\frac{1}{p}} .
$$

This completes the proof in case of (B) with $k_{r}=0$.

Step 5: We show (3.11) in case (C), i.e. for $p \in[1, \infty)$ let $\mathcal{U}_{0}=\mathcal{Y}_{0}=C W_{0}^{\infty, p}\left(\mathbb{R}_{\geq 0} \rightarrow \mathbb{R}\right)$. For brevity write $\|\cdot\|_{L^{p}}:=\|\cdot\|_{L^{p}\left(\mathbb{R}_{>0} \rightarrow \mathbb{R}\right)}$. Since $y^{(i+1)} \in C W_{0}^{1, p}\left(\mathbb{R}_{\geq 0} \rightarrow \mathbb{R}\right)$, it follows from Proposition 3.2 and inequality (3.14), that for all $y \in \mathcal{Y}_{0}$ and $i \in\{1, \ldots, r-1\}$

$$
\begin{aligned}
\left\|\Delta_{h}^{i}(y)-y^{(i)}\right\|_{\mathcal{U}_{0}} & \leq i h\left\|M_{i h}\left[y^{(i+1)}\right](\cdot)\right\|_{\mathcal{U}_{0}} \\
& \leq i h \sum_{\mu=0}^{\infty}\left[2\left\|y^{(\mu+i+1)}\right\|_{L^{p}}^{p-1}\left(\left\|y^{(\mu+i+1)}\right\|_{L^{p}}+i h p\left\|y^{(\mu+i+2)}\right\|_{L^{p}}\right)\right]^{\frac{1}{p}} \\
& \leq 2^{\frac{1}{p}} i h\left(\sum_{\mu=0}^{\infty}\left\|y^{(\mu+i+1)}\right\|_{L^{p}}+i h p \sum_{\mu=0}^{\infty}\left\|y^{(\mu+i+1)}\right\|_{L^{p}}^{\frac{p-1}{p}}\left\|y^{(\mu+i+2)}\right\|_{L^{p}}^{\frac{1}{p}}\right) \\
& \leq 2^{\frac{1}{p}} i h\left(\sum_{\mu=0}^{\infty}\left\|y^{(\mu+i+1)}\right\|_{L^{p}}+i h p\left(\sum_{\mu=0}^{\infty}\left\|y^{(\mu+i+1)}\right\|_{L^{p}}^{\frac{p-1}{p}}\right)\left(\sum_{\mu=0}^{\infty}\left\|y^{(\mu+i+2)}\right\|_{L^{p}}^{\frac{1}{p}}\right)\right) \\
& \leq 2^{\frac{1}{p}} i h\left(\sum_{\mu=0}^{\infty}\left\|y^{(\mu)}\right\|_{L^{p}}+i h p\left(\sum_{\mu=0}^{\infty}\left\|y^{(\mu)}\right\|_{L^{p}}^{\frac{p-1}{p}}\right)\left(\sum_{\mu=0}^{\infty}\left\|y^{(\mu)}\right\|_{L^{p}}^{\frac{1}{p}}\right)\right) \\
& \leq 2^{\frac{1}{p}} i h(1+i h p) \sum_{\mu=0}^{\infty}\left\|y^{(\mu)}\right\|_{L^{p}} \\
& =2^{\frac{1}{p}} i h(1+i h p)\|y\|_{\mathcal{Y}_{0}}
\end{aligned}
$$

and so (3.13) yields

$$
\vec{\delta}\left(C_{k}, C_{k}^{\text {Euler }}[h]\right) \leq 2^{\frac{1}{p}} h \sum_{i=0}^{r-1}\left|k_{i+1}\right| \cdot i(1+i h p) .
$$

which completes the proof in case of $(\mathrm{C})$ and concludes the proof of the theorem.

\section{Applications to linear minimum phase systems}

The main result, Theorem 3.1, is stated for various signal spaces (3.2). We now consider linear systems in detail to illustrate how the choice of signal space is determined by relative degree assumptions on the linear system and the stabilizability requirements in the various signal 
spaces. In particular we consider the class $\mathcal{P}_{n, r}$ of all state space triples $(A, b, c)$ corresponding to $n$-dimensional, minimum phase, single-input, single-output systems with relative degree $r \in\{1, \ldots, n\}$ and positive high frequency gain $c A^{r-1} b$. Let $(A, b, c) \in \mathcal{P}_{n, r}, x^{0} \in \mathbb{R}^{n}$ and $P\left(A, b, c ; x^{0}\right): \mathcal{U}_{e} \rightarrow \mathcal{Y}_{e}$ be the associated plant operator $u_{1} \mapsto y_{1}$ given by

$$
\left.\begin{array}{rl}
\dot{x} & =A x+b u_{1}, \quad x(0)=x^{0} \\
y_{1} & =c x,
\end{array}\right\},
$$

where $\mathcal{U}$ and $\mathcal{Y}$ are any of the input/output signal spaces pairs given in (3.2). We establish stability properties for both the nominal closed-loop system $\left[P\left(A, b, c ; x^{0}\right), C_{k}\right]$ and the closedloop system with the delay based controller $\left[P\left(A, b, c ; x^{0}\right), C_{k}^{\text {Euler }}[h]\right]$. In particular, we show exponential stability of the initial value problems for closed-loop systems with zero disturbances $u_{0} \equiv y_{0} \equiv 0$ and gain stability of the closed-loop systems with arbitrary $u_{0}, y_{0}$ from signal spaces in cases (A), (B) or (C). For the following consider the high-gain control design:

$$
C_{k, \kappa, \nu}: \mathcal{Y}_{e} \rightarrow \mathcal{U}_{e}, \quad y_{2} \mapsto u_{2}=-\nu \sum_{i=0}^{r-1} \kappa^{r-i} k_{i+1} y_{2}^{(i)},
$$

where $\kappa, \nu \geq 1$ are suitably large scalars which are to be determined and $k=\left(k_{1}, \ldots k_{r}\right)$ is such that $k_{r}>0$ and the polynomial $s \mapsto \sum_{i=0}^{r-1} k_{i+1} s^{i}$ is Hurwitz, i.e. has all roots in $\mathbb{C}_{-}$.

\subsection{Exponential stability of $\left[P\left(A, b, c ; x^{0}\right), C_{k, \kappa, \nu}\right]$ with $u_{0} \equiv y_{0} \equiv 0$}

In Proposition 4.1 we present how the high-gain derivative feedback controller of form (4.2) stabilizes systems $(A, b, c) \in \mathcal{P}_{n, r}, r \leq n$, given by (4.1). We show that there exist $\kappa, \nu \geq 1$ such that an application of controller $C_{k, \kappa, \nu}$ to a linear system $(A, b, c)$ yields an exponentially stable closed-loop system $\left[P\left(A, b, c ; x^{0}\right), C_{k, \kappa, \nu}\right]$ with $u_{0} \equiv y_{0} \equiv 0$.

With controller $C_{k, \kappa, \nu}$ we use a static feedback of derivatives of the output signal to stabilize linear systems. Note that there are only structural conditions to the considered system $(A, b, c)$ : the relative degree is known, the system is minimum phase and has a positive high-frequency gain $c A^{r-1} b$.

Proposition 4.1 Let, for $r, n \in \mathbb{N}$ with $r \leq n,(A, b, c) \in \mathcal{P}_{n, r}$ and $x^{0} \in \mathbb{R}^{n}$. Suppose $k=\left(k_{1}, \ldots, k_{r}\right) \in \mathbb{R}^{1 \times r}$ with $k_{r}>0$ and $s \mapsto \sum_{i=0}^{r-1} k_{i+1} s^{i}$ Hurwitz. Then, for sufficiently large $\kappa, \nu \geq 1$, the closed-loop system $\left[P\left(A, b, c ; x^{0}\right), C_{k, \kappa, \nu}\right]$ given by (4.1), (4.2), (2.1) with $u_{0} \equiv y_{0} \equiv 0$ is exponentially stable, in the sense

$\exists \nu^{*} \geq 1 \forall \nu \geq \nu^{*} \exists \kappa^{*} \geq 1 \exists M>0 \exists \alpha>0 \forall \kappa \geq \kappa^{*} \forall t \geq 0 \forall x^{0} \in \mathbb{R}^{n}:\left|x\left(t ; x^{0}\right)\right| \leq M e^{-\alpha t}\left|x^{0}\right|$, where $x\left(\cdot ; x^{0}\right)$ denotes the solution of (4.1), (4.2), (2.1) with $u_{0} \equiv y_{0} \equiv 0$.

Proposition 4.1 shows the existence of parameters $\kappa, \nu \geq 1$ with which controller (4.2) stabilizes system (4.1). Explicit bounds for $\kappa$ and $\nu$, which are not given here, depend only on the system matrices $A, b, c$ and the vector $k=\left(k_{1}, \ldots, k_{r}\right)$.

The proof of Proposition 4.1 is based on the following Byrnes-Isidori normal form.

Lemma 4.2 Let, for $r, n \in \mathbb{N}$ with $r \leq n,(A, b, c) \in \mathcal{P}_{n, r}$. For $\mathcal{C}:=\left[c / c A / \ldots / c A^{r-1}\right]$, $\mathcal{B}:=\left[b, A b, \ldots, A^{r-1} b\right], \mathcal{N} \in \mathbb{R}^{n \times(n-r)}$ with $\operatorname{im} \mathcal{N}=\operatorname{ker} \mathcal{C}$ and

$$
V=\left[\begin{array}{c}
\mathcal{C} \\
\left(\mathcal{N}^{T} \mathcal{N}\right)^{-1} \mathcal{N}^{T}\left[I_{n-r}-\mathcal{B}(\mathcal{C B})^{-1} \mathcal{C}\right]
\end{array}\right], \quad V^{-1}=\left[\mathcal{B}(\mathcal{C B})^{-1}, \mathcal{N}\right]
$$


the coordinate transformation

$$
\left(\begin{array}{l}
\xi \\
\eta
\end{array}\right)(t):=V x(t)
$$

converts $(A, b, c)$, given by (4.1) into the normal form $\left(V A V^{-1}, V b, c V^{-1}\right) \in \mathcal{P}_{n, r}$ :

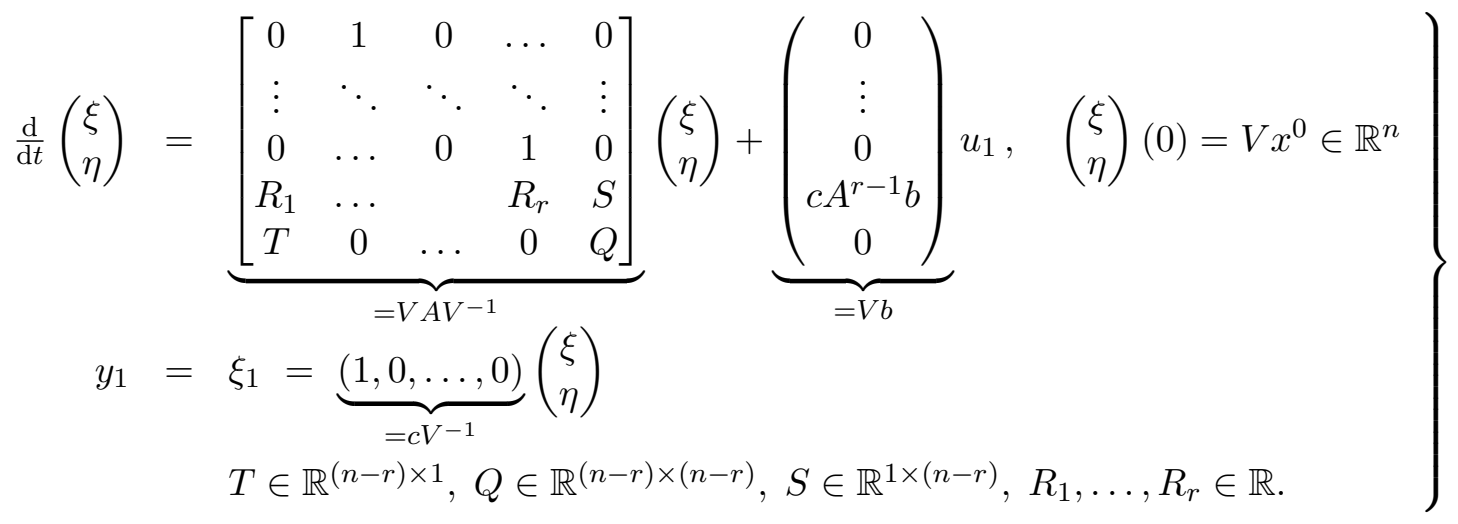

Proof. See [9, Lem. 3.5].

Remark 4.3 Proposition 4.1 shows that for every system $(A, b, c) \in \mathcal{P}_{n, r}$ of form $(4.1), r, n \in \mathbb{N}$ with $r \leq n$, we may choose $k \in \mathbb{R}^{1 \times r}$ such that

$$
\operatorname{spec}\left(A+b k\left[c / \ldots / c A^{r-1}\right]\right)=\operatorname{spec}\left(V A V^{-1}+V b(k \mid 0)\right) \subset \mathbb{C}_{-},
$$

where the transformation matrix $V \in \mathbb{R}^{n \times n}$ is given by $(4.3), k=\left(k_{1}, \ldots, k_{r}\right)$ with $k_{i}:=$ $\nu \kappa^{r+1-i} \widetilde{k}_{i}, i \in\{1, \ldots, r\}, s \mapsto \sum_{i=0}^{r-1} \widetilde{k}_{i+1} s^{i}$ with $\widetilde{k}_{r}>0$ is any Hurwitz polynomial and $\kappa, \nu \geq 1$ are sufficiently large.

For the following results in case of signal spaces of type (B) in (3.2) we assume that $k_{r}=0$ and $\operatorname{spec}\left(A+b k\left[c / \ldots / c A^{r-1}\right]\right) \subset \mathbb{C}_{-}$. In this case we cannot refer to Propostion 4.1.

Proof of Proposition 4.1. By equations (2.1) and (4.2),

$$
u_{1}(t)=-\sum_{i=0}^{r-1} \nu \kappa^{r-i} k_{i+1}\left[y_{1}^{(i)}(t)-y_{0}^{(i)}(t)\right]+u_{0}(t) .
$$

The closed-loop system (4.1), (4.6) is equivalent to (4.5), (4.6). Setting $\zeta_{i}=\kappa^{-i+1} \xi_{i}$, for $i=$ $1, \ldots, r$, yields

$$
\begin{aligned}
\dot{\zeta}_{i} & =\kappa^{-i+1} \dot{\xi}_{i}=\kappa^{-i+1} \xi_{i+1}=\kappa \zeta_{i+1}, \quad \text { for } i=1, \ldots, r-1, \\
\dot{\zeta}_{r} & =\kappa^{-r+1} \dot{\xi}_{r} \\
& =\kappa^{-r+1}\left(\left(R_{1}-\vartheta \nu k_{1} \kappa^{r}\right) \xi_{1}+\cdots+\left(R_{r-1}-\vartheta \nu k_{r-1} \kappa^{2}\right) \xi_{r-1}+\left(R_{r}-\vartheta \nu k_{r} \kappa\right) \xi_{r}\right)+\kappa^{-r+1} S \eta \\
& =\kappa\left(\left(\frac{R_{1}}{\kappa^{r}}-\vartheta \nu k_{1}\right) \zeta_{1}+\cdots+\left(\frac{R_{r-1}}{\kappa^{2}}-\vartheta \nu k_{r-1}\right) \zeta_{r-1}+\left(\frac{R_{r}}{\kappa}-\vartheta \nu k_{r}\right) \zeta_{r}\right)+\kappa^{-r+1} S \eta .
\end{aligned}
$$

Thus the scaling

$$
\left(\begin{array}{l}
\zeta \\
\eta
\end{array}\right)=U_{\kappa}\left(\begin{array}{l}
\xi \\
\eta
\end{array}\right), \quad U_{\kappa}:=\operatorname{diag}\left(1, \kappa^{-1}, \ldots, \kappa^{-r+1}, 1, \ldots, 1\right),
$$


converts (4.5), (4.6) into

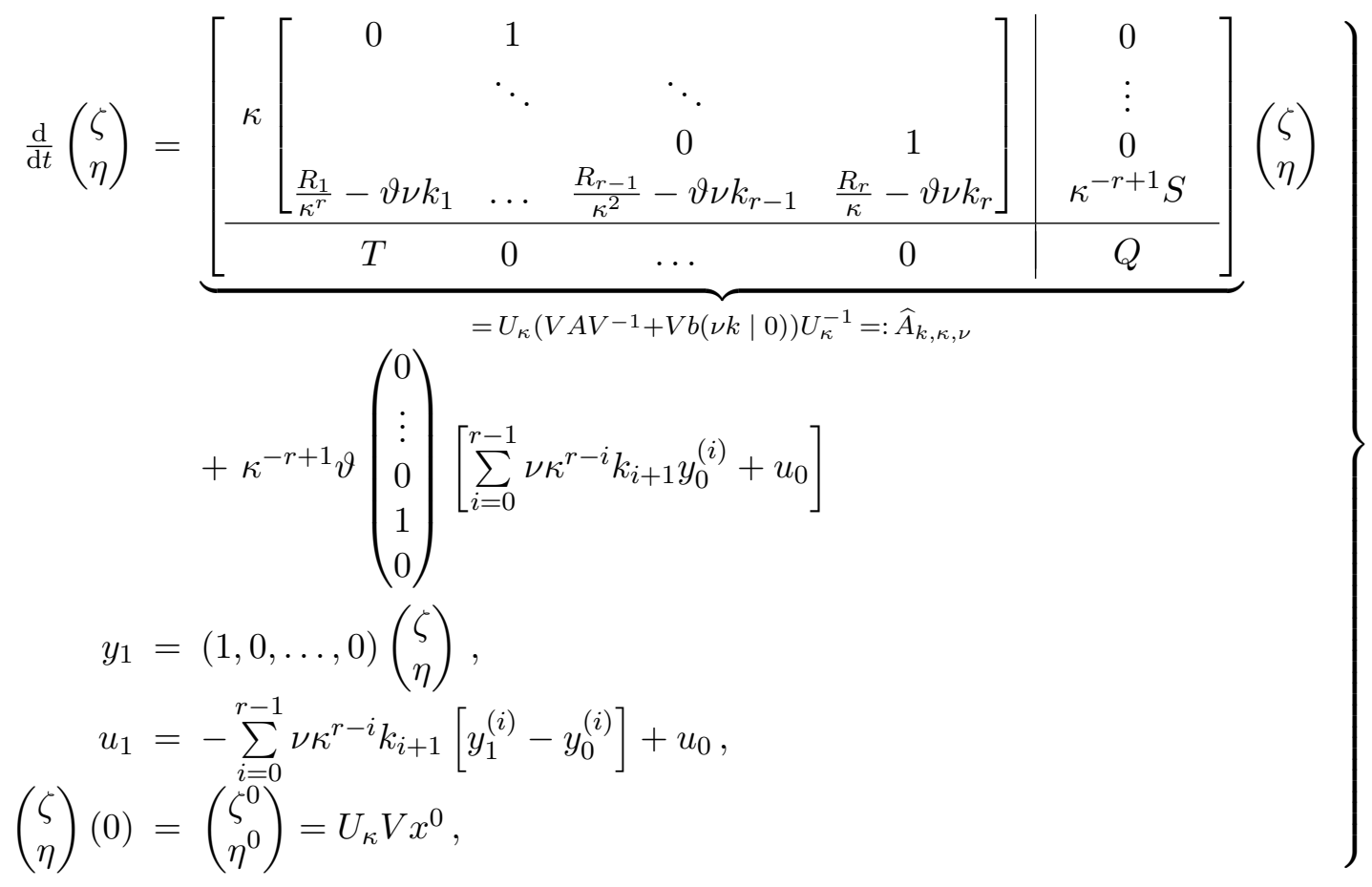

where $\vartheta=c A^{r-1} b, R_{1}, \ldots, R_{r} \in \mathbb{R}, S \in \mathbb{R}^{1 \times(n-r)}, T \in \mathbb{R}^{n-r}, Q \in \mathbb{R}^{(n-r) \times(n-r)}$ with $\operatorname{spec}(Q) \subset$ $\mathbb{C}_{-}$and $V \in \mathbb{R}^{n \times n}$ with $\operatorname{det} V \neq 0$.

In view of $u_{0} \equiv y_{0} \equiv 0$ and the equivalence of (4.1), (4.2), (2.1) and the closed-loop equations (4.7) it remains to show that

$$
\exists \nu^{*} \geq 1 \forall \nu \geq \nu^{*} \exists \kappa^{*} \geq 1 \exists M>0 \exists \alpha>0 \quad \forall \kappa \geq \kappa^{*} \forall t \geq 0:\left|e^{\widehat{A}_{k, \kappa, \nu} t}\right| \leq M e^{-\alpha t} .
$$

Since $k_{r}>0, \vartheta>0$ and $s \mapsto p(s)=\sum_{i=0}^{r-1} k_{i+1} s^{i}$ is Hurwitz, it follows from the root-locus [14, Sect. 5] that $r-1$ roots of $q_{\nu}(s)=s^{r}+\nu \vartheta p(s)=0$ converge to the roots of $p(s)=0$ as $\nu \rightarrow \infty$ and that the remaining root of $q_{\nu}$ is real and diverges to $-\infty$ as $\nu \rightarrow \infty$. Thus there exists $\nu^{*}>0$ such that the polynomial $q_{\nu}$ is Hurwitz, for all $\nu \geq \nu^{*}$. Let $\nu \geq \nu^{*}$ and choose the positive definite matrices $N_{\zeta}=N_{\zeta}^{T} \in \mathbb{R}^{r \times r}$ and $N_{\eta}=N_{\eta}^{T} \in \mathbb{R}^{(n-r) \times(n-r)}$ solving

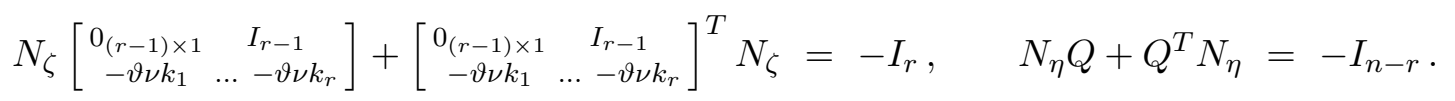

Then the derivative of

$$
t \mapsto V(t):=\frac{1}{2} \zeta(t)^{T} N_{\zeta} \zeta(t)+\frac{1}{2} \eta(t)^{T} N_{\eta} \eta(t)
$$

along the solution of

$$
\frac{\mathrm{d}}{\mathrm{d} t}\left(\begin{array}{l}
\zeta \\
\eta
\end{array}\right)(t)=\widehat{A}_{k, \kappa, \nu}\left(\begin{array}{l}
\zeta \\
\eta
\end{array}\right)(t)
$$


yields, for all $t \geq 0$, and omitting the argument $t$ for brevity,

$$
\begin{aligned}
& \dot{V}(t)=\frac{\mathrm{d}}{\mathrm{d} t}\left(\frac{1}{2} \zeta^{T} N_{\zeta} \zeta+\frac{1}{2} \eta^{T} N_{\eta} \eta\right) \\
& =\zeta^{T} N_{\zeta}\left(\kappa\left[\begin{array}{cccc}
0 & 1 & & \\
& \ddots & \ddots & \\
& & 0 & 1 \\
\frac{R_{1}}{\kappa^{r}}-\vartheta \nu k_{1} & \ldots & \frac{R_{r-1}}{\kappa^{2}}-\vartheta \nu k_{r-1} & \frac{R_{r}}{\kappa}-\vartheta \nu k_{r}
\end{array}\right] \zeta+\left[\begin{array}{c}
0 \\
\vdots \\
\kappa^{-r+1} S \eta
\end{array}\right]\right)+\eta^{T} N_{\eta}\left(Q \eta+T \zeta_{1}\right) \\
& \stackrel{(4.9)}{\leq}-\frac{\kappa}{2}|\zeta|^{2}+\kappa \zeta N_{\zeta}\left[\begin{array}{ccc}
0 & & \\
& \ddots & \\
\frac{R_{1}}{\kappa^{r}} & \ldots & \frac{R_{r}}{\kappa}
\end{array}\right] \zeta+\frac{1}{\kappa^{r-1}}\left|N_{\zeta}\right||S||\zeta||\eta|-\frac{1}{2}|\eta|^{2}+\left|N_{\eta} T\right||\eta|\left|\zeta_{1}\right| \\
& \stackrel{\kappa \geq 1}{\leq}-\frac{\kappa}{2}|\zeta|^{2}+\left|N_{\zeta}\right|\left|\left(R_{1}, \ldots, R_{r}\right)\right||\zeta|^{2}+\frac{1}{\kappa^{r-1}}\left|N_{\zeta}\right||S||\zeta|^{2}+\frac{1}{\kappa^{r-1}}\left|N_{\zeta}\right||S||\eta|^{2} \\
& -\frac{1}{2}|\eta|^{2}+\frac{1}{4}|\eta|^{2}+4\left|N_{\eta} T\right|\left|\zeta_{1}\right|^{2} \\
& \leq-\left(\frac{\kappa}{2}-\left|N_{\zeta}\right|\left|\left(R_{1}, \ldots, R_{r}\right)\right|-\left|N_{\zeta}\right||S|-4\left|N_{\eta} T\right|\right)|\zeta|^{2}-\left(\frac{1}{4}-\frac{\left|N_{\zeta}\right||S|}{\kappa^{r-1}}\right)|\eta|^{2},
\end{aligned}
$$

and so, for

$$
\begin{aligned}
\kappa^{*} & :=\max \left\{\frac{1}{4}+2\left(\left|N_{\zeta}\right|\left|\left(R_{1}, \ldots, R_{r}\right)\right|-\left|N_{\zeta}\right||S|-4\left|N_{\eta} T\right|\right),\left(8\left|N_{\zeta}\right||S|\right)^{-r+1}\right\}, \\
\alpha & :=\min \left\{\frac{1}{8\left|N_{\zeta}\right|}, \frac{1}{8\left|N_{\eta}\right|}\right\},
\end{aligned}
$$

we conclude, for all $t \geq 0$ and $\kappa \geq \kappa^{*}$,

$$
\dot{V}(t) \leq-\frac{1}{8}|\zeta(t)|^{2}-\frac{1}{8}|\eta(t)|^{2} \leq-\frac{1}{8\left|N_{\zeta}\right|} \zeta(t)^{T} N_{\zeta} \zeta(t)-\frac{1}{8\left|N_{\eta}\right|} \eta(t)^{T} N_{\eta} \eta(t) \leq-\alpha V(t),
$$

hence

$$
\forall t \geq t_{0} \forall t_{0} \geq 0:\left|\left(\begin{array}{l}
\zeta(t) \\
\eta(t)
\end{array}\right)\right| \leq \exp \left(-\alpha\left(t-t_{0}\right)\right) \sqrt{\frac{\operatorname{maxspec}\left[\begin{array}{cc}
N_{\zeta} & 0 \\
0 & N_{\eta}
\end{array}\right]}{\operatorname{minspec}\left[\begin{array}{cc}
N_{\zeta} & 0 \\
0 & N_{\eta}
\end{array}\right]}}\left|\left(\begin{array}{l}
\zeta\left(t_{0}\right) \\
\eta\left(t_{0}\right)
\end{array}\right)\right| .
$$

This proves (4.8) and completes the proof of the proposition.

\subsection{Stability properties of the closed-loop system $\left[P\left(A, b, c ; x^{0}\right), C_{k}\right]$}

Now we show for $(A, b, c) \in \mathcal{P}_{n, r}$ with $k \in \mathbb{R}^{1 \times r}$ such that $\operatorname{spec}\left(A+b k\left[c / \ldots / c A^{r-1}\right]\right) \subset \mathbb{C}_{-}$ and for appropriate input/output signal spaces of types (A), (B) or (C) in (3.2) that if $x^{0}=0$, then the closed-loop system $\left[P\left(A, b, c ; x^{0}\right), C_{k}\right]$ is gain stable on $\mathcal{W}_{0}$. For the input/output signal spaces of type (A) or (B) only, we also show that the closed-loop system $\left[P\left(A, b, c ; x^{0}\right), C_{k}\right]$ is $\mathcal{W}$-stable for any initial conditions $x^{0} \in \mathbb{R}^{n}$.

Theorem 4.4 Let, for $r, n \in \mathbb{N}$ with $r \leq n,(A, b, c) \in \mathcal{P}_{n, r}$ given by (4.1) and choose $k \in \mathbb{R}^{1 \times r}$ such that $\operatorname{spec}\left(A+b k\left[c / \ldots / c A^{r-1}\right]\right) \subset \mathbb{C}_{-}$. Let the signal spaces $\mathcal{U}, \mathcal{Y}, \mathcal{W}, \mathcal{W}_{0}$ be of type (A), (B) or (C) in (3.2); in case of (B) suppose $k e_{r}^{(r)}=0$. Consider the controller operator $C_{k}: \mathcal{Y}_{e} \rightarrow \mathcal{U}_{e}$, as defined by (1.1) and the associated plant operator $P\left(A, b, c ; x^{0}\right): \mathcal{U}_{e} \rightarrow \mathcal{Y}_{e}$ with initial value $x^{0} \in \mathbb{R}^{n}$ as defined by (4.1). Then the closed-loop system $\left[P(A, b, c ; 0), C_{k}\right]$ is $\mathcal{W}_{0}$-gain stable. In the case of signal spaces given by $(\mathrm{A})$ or $(\mathrm{B})$, the closed-loop system $\left[P\left(A, b, c ; x^{0}\right), C_{k}\right]$ is also $\mathcal{W}$-stable. 
Proof. Step 1: Consider $\mathcal{W}$ of type $(\mathrm{A}),(\mathrm{B})$ or $(\mathrm{C})$ given by $(3.2)$ and let $\left(u_{0}, y_{0}\right) \in \mathcal{W}$. The closed-loop system $\left[P\left(A, b, c ; x^{0}\right), C_{k}\right]$ given by equations $(4.1),(1.1),(2.1)$ is, in view of coordinate transformation (4.4), equivalent to (4.5), (1.1), (2.1). Invoking Lemma 4.2 and applying Variation of Constants yields

$$
\forall t \geq 0:\left(\begin{array}{l}
\xi \\
\eta
\end{array}\right)(t)=e^{V\left(A+b k\left[\begin{array}{c}
c \\
\vdots \\
c A^{r-1}
\end{array}\right]\right) V^{-1} t}\left(\begin{array}{c}
\xi^{0} \\
\eta^{0}
\end{array}\right)+\int_{0}^{t} e^{V\left(A+b k\left[\begin{array}{c}
c \\
\vdots \\
c A^{r-1}
\end{array}\right]\right) V^{-1}(t-s)} \varphi(s) \mathrm{d} s,
$$

where, in view of $u_{0} \in \mathcal{U}$ and $y_{0} \in \mathcal{Y}$,

$$
\varphi(\cdot):=\left(\begin{array}{c}
0_{r-1} \\
c A^{r-1} b \\
0_{n-r}
\end{array}\right)\left[u_{0}(\cdot)+\left(C_{k} y_{0}\right)(\cdot)\right] \in \mathcal{U}
$$

Step 2: Consider case (A) or (B), i.e. $\mathcal{U} \times \mathcal{Y}=C L^{p}\left(\mathbb{R}_{\geq 0} \rightarrow \mathbb{R}\right) \times C W^{r, p}\left(\mathbb{R}_{\geq 0} \rightarrow \mathbb{R}\right), 1 \leq p<\infty$, $r \leq n$. Taking norms in (4.10) and invoking the well-known inequality $\left\|\int_{0}^{-} f(\cdot-s) g(s) \mathrm{d} s\right\|_{L^{p}} \leq$ $\|f\|_{L^{1}}\|g\|_{L^{p}}$, for $f \in L^{1}$ and $g \in L^{p}$, we obtain, for some $\beta_{1}, \beta_{2}>0$,

$$
\begin{aligned}
\left\|\left(\begin{array}{c}
\xi \\
\eta
\end{array}\right)\right\|_{L^{p}\left(\mathbb{R}_{\geq 0} \rightarrow \mathbb{R}^{n}\right)} & \leq \beta_{1}\left[\left|\left(\begin{array}{c}
\xi^{0} \\
\eta^{0}
\end{array}\right)\right|+\|\varphi\|_{L^{p}\left(\mathbb{R}_{\geq 0} \rightarrow \mathbb{R}^{n}\right)}\right] \\
& \leq \beta_{1}\left|\left(\begin{array}{c}
\xi^{0} \\
\eta^{0}
\end{array}\right)\right|+\beta_{1} \beta_{2}\left[\left\|u_{0}\right\|_{L^{p}\left(\mathbb{R}_{\geq 0} \rightarrow \mathbb{R}\right)}+\sum_{i=0}^{r-1}\left|k_{i+1}\right|\left\|y_{0}^{(i)}\right\|_{L^{p}\left(\mathbb{R}_{\geq 0} \rightarrow \mathbb{R}\right)}\right]
\end{aligned}
$$

and thus,

$$
\left(\begin{array}{l}
\xi \\
\eta
\end{array}\right) \in L^{p}\left(\mathbb{R}_{\geq 0} \rightarrow \mathbb{R}^{n}\right)
$$

Now, by (4.5),

$$
\begin{array}{lc}
y_{1}^{(i)}=\xi_{i+1} \in L^{p}\left(\mathbb{R}_{\geq 0} \rightarrow \mathbb{R}\right), & \text { for } i=0, \ldots, r-1 \\
y_{1}^{(r)}=\dot{\xi}_{r}=\left(\sum_{i=1}^{r}\left(R_{i}-c A^{r-1} b k_{i}\right) \xi_{i}\right)+S \eta+\varphi \in L^{p}\left(\mathbb{R}_{\geq 0} \rightarrow \mathbb{R}\right)
\end{array}
$$

and with (4.11) it follows that $y_{1} \in C W^{r, p}\left(\mathbb{R}_{\geq 0} \rightarrow \mathbb{R}\right)=\mathcal{Y}$.

Finally,

$$
u_{1}=u_{0}-C_{k}\left(y_{2}\right)=u_{0}-C_{k}\left(y_{0}\right)+C_{k}\left(y_{1}\right) \in C L^{p}\left(\mathbb{R}_{\geq 0} \rightarrow \mathbb{R}\right)=\mathcal{U},
$$

and we have shown that the closed-loop system $\left[P\left(A, b, c ; x^{0}\right), C_{k}\right]$ is $\mathcal{W}$-stable in case (A) and (B).

Step 3: Let $x^{0}=0$ and let $\mathcal{W}_{0}$ be as in (A) or (B), i.e. $\mathcal{U}_{0} \times \mathcal{Y}_{0}=C L^{p}\left(\mathbb{R}_{\geq 0} \rightarrow \mathbb{R}\right) \times C W_{0}^{r, p}\left(\mathbb{R}_{\geq 0} \rightarrow\right.$ $\mathbb{R}), 1 \leq p<\infty, r \leq n$. It is straightforward to see that $y^{(i)}(0)=0$ for $i=0, \ldots, r$, and hence one can show similarly as in Step 2 that, for some $\beta_{1}, \ldots, \beta_{5} \geq 1$,

$$
\begin{aligned}
\left\|y_{1}\right\|_{C W_{0}^{r, p}\left(\mathbb{R}_{\geq 0} \rightarrow \mathbb{R}\right)} & \leq \beta_{1} \beta_{2} \beta_{3}\left[\left\|u_{0}\right\|_{C L^{p}\left(\mathbb{R}_{\geq 0} \rightarrow \mathbb{R}\right)}+\sum_{i=0}^{r-1}\left|k_{i+1}\right|\left\|y_{0}^{(i)}\right\|_{C L^{p}\left(\mathbb{R}_{\geq 0} \rightarrow \mathbb{R}\right)}\right] \\
& \leq \beta_{4}\left[\left\|u_{0}\right\|_{C L^{p}\left(\mathbb{R}_{\geq 0} \rightarrow \mathbb{R}\right)}+\left\|y_{0}\right\|_{C W_{0}^{r-1, p}\left(\mathbb{R}_{\geq 0} \rightarrow \mathbb{R}\right)}\right]
\end{aligned}
$$

and

$$
\begin{aligned}
\left\|u_{1}\right\|_{C L^{p}\left(\mathbb{R}_{\geq 0} \rightarrow \mathbb{R}\right)} & \leq\left\|u_{0}\right\|_{C L^{p}\left(\mathbb{R}_{\geq 0} \rightarrow \mathbb{R}\right)}+\left\|C_{k} y_{2}\right\|_{C L^{p}\left(\mathbb{R}_{\geq 0} \rightarrow \mathbb{R}\right)} \\
& \leq\left\|u_{0}\right\|_{C L^{p}\left(\mathbb{R}_{\geq 0} \rightarrow \mathbb{R}\right)}+\beta_{5}\left\|y_{2}\right\|_{C W_{0}^{r-1, p}\left(\mathbb{R}_{\geq 0} \rightarrow \mathbb{R}\right)} \\
& \leq\left\|u_{0}\right\|_{C L^{p}\left(\mathbb{R}_{\geq 0} \rightarrow \mathbb{R}\right)}+\beta_{5}\left[\left\|y_{1}\right\|_{C W_{0}^{r-1, p}\left(\mathbb{R}_{\geq 0} \rightarrow \mathbb{R}\right)}+\left\|y_{0}\right\|_{C W_{0}^{r-1, p}\left(\mathbb{R}_{\geq 0} \rightarrow \mathbb{R}\right)}\right]
\end{aligned}
$$


and thus $\mathcal{W}_{0}$ gain stability in case (A), (B) follows.

Step 4: Let $x^{0}=0$ and let $\mathcal{W}_{0}$ be as in $(\mathrm{C})$, i.e. $\mathcal{U}_{0}=\mathcal{Y}_{0}=C W_{0}^{\infty, p}\left(\mathbb{R}_{\geq 0} \rightarrow \mathbb{R}\right), 1 \leq p<\infty$. First note that $\varphi \in C W_{0}^{\infty, p}\left(\mathbb{R}_{\geq 0} \rightarrow \mathbb{R}\right)$. By [17, Proposition VI.3.1] we have, for all $i \in \mathbb{N}$ and $t \geq 0$

$$
\frac{\mathrm{d}^{i}}{\mathrm{~d} t^{i}} \int_{0}^{t} e^{V\left(A+b k\left[\begin{array}{c}
c \\
\vdots \\
c A^{r-1}
\end{array}\right]\right) V^{-1}(t-s)} \varphi(s) \mathrm{d} s=\int_{0}^{t} e^{V\left(A+b k\left[\begin{array}{c}
c \\
\vdots \\
c A^{r-1}
\end{array}\right]\right) V^{-1}(t-s)} \varphi^{(i)}(s) \mathrm{d} s .
$$

Hence it follows from (4.10) that $\left.\frac{\mathrm{d}^{i}}{\mathrm{~d} t^{i}}\left(\begin{array}{l}\xi \\ \eta\end{array}\right)\right|_{t=0}=0$, and so $y^{(i)}(0)=0$ for all $i \in \mathbb{N}$. It follows also that $u_{1}^{(i)}(0)=u_{0}^{(i)}(0)-C_{k}\left(y_{0}^{(i)}(0)\right)+C_{k}\left(y_{1}^{(i)}(0)\right)=0$ for all $i \in \mathbb{N}$. One can then show similarly as in Step 3 that, for some $\beta_{1}, \beta_{2} \geq 1$,

$$
\begin{aligned}
\left\|y_{1}\right\|_{C W^{\infty, p}\left(\mathbb{R}_{\geq 0} \rightarrow \mathbb{R}\right)} & =\sum_{j \geq 0}\left\|y_{1}\right\|_{C L^{p}\left(\mathbb{R}_{\geq 0} \rightarrow \mathbb{R}\right)} \\
& \leq \beta_{1} \sum_{j \geq 0}\left[\left\|u_{0}^{(j)}\right\|_{C L^{p}\left(\mathbb{R}_{\geq 0} \rightarrow \mathbb{R}\right)}+\left\|\left(C_{k} y_{0}\right)^{(j)}\right\|_{C L^{p}\left(\mathbb{R}_{\geq 0} \rightarrow \mathbb{R}\right)}\right] \\
& \leq \beta_{1}\left\|u_{0}\right\|_{C W^{\infty, p}\left(\mathbb{R}_{\geq 0} \rightarrow \mathbb{R}\right)}+\beta_{2} \sum_{j \geq 0} \sum_{i=0}^{r-1}\left\|y_{0}^{(i+j)}\right\|_{C L^{p}\left(\mathbb{R}_{\geq 0} \rightarrow \mathbb{R}\right)} \\
& \leq \beta_{1}\left\|u_{0}\right\|_{C W^{\infty, p}\left(\mathbb{R}_{\geq 0} \rightarrow \mathbb{R}\right)}+r \beta_{2}\left\|y_{0}\right\|_{C W^{\infty, p}\left(\mathbb{R}_{\geq 0} \rightarrow \mathbb{R}\right)} .
\end{aligned}
$$

An analogous inequality for $\left\|u_{1}\right\|_{C W^{\infty, p}\left(\mathbb{R}_{\geq 0} \rightarrow \mathbb{R}\right)}$ gives $\mathcal{W}_{0}$ gain stability as required. This completes the proof of the theorem.

Theorem 4.4 shows in combination with Proposition 4.1, for signal spaces of type (A) or (C) in (3.2), that if $r \leq n$ and $\kappa, \nu \geq 1$ sufficiently large then $\left[P\left(A, b, c ; x^{0}\right), C_{k, \kappa, \nu}\right]$ is $\mathcal{W}_{0}$-gain stable, with a bound for the gain given by

$$
\left\|\Pi_{C_{k, \kappa, \nu} / / P(A, b, c ; 0)}\right\|_{\mathcal{W}_{0}, \mathcal{W}_{0}} \leq \beta(k, \kappa, \nu),
$$

for some $\beta(k, \kappa, \nu)>0$ determined by the proof of Theorem 4.4 and Proposition 4.1.

In the signal space setting of type (B) in (3.2), i.e. $p, r<\infty$, these stability results are only proved for $k_{r}=0$, thus precluding the application of Proposition 4.1. However, there are many plants stabilizable in $\mathcal{P}_{n, r}, r \leq n-1$, including, for example, the class of plants stabilizable by PD controllers $(r=2, n \geq 3)$.

Since Proposition 4.1 gives stabilizability of plants in $\mathcal{P}_{n, r}, r \leq n$, and since the signal space setting (A) is only applicable when $p=\infty$, the setting (C) has been introduced to allow stability results in the context of $p<\infty$, without the assumption that $k_{r}=0$ as in (B). However, the setting $(\mathrm{C})$ does introduce extra regularity requirements on the external disturbances $u_{0}, y_{0}$.

\subsection{Gain stability of the closed-loop system $\left[P(A, b, c ; 0), C_{k}^{\text {Euler }}[h]\right]$}

We are now in a position to show that linear systems $(A, b, c) \in \mathcal{P}_{n, r}$ are gain stabilizable on $\mathcal{U} \times \mathcal{Y}$ by the delay feedback $C_{k}^{\text {Euler }}[h]$ defined in (1.2), for suitable $k \in \mathbb{R}^{1 \times r}$, for sufficiently small $h>0$ and for signal spaces of type (A), (B) or (C) in (3.2).

Theorem 4.5 Let, for $r, n \in \mathbb{N}$ with $r \leq n,(A, b, c) \in \mathcal{P}_{n, r}$ and choose $k \in \mathbb{R}^{1 \times r}$ such that $\operatorname{spec}\left(A+b k\left[c / \ldots / c A^{r-1}\right]\right) \subset \mathbb{C}_{-}$. Let the signal spaces $\mathcal{U}, \mathcal{Y}, \mathcal{W}, \mathcal{W}_{0}$ be of type (A), (B) or 
(C) in (3.2); in case of (B) suppose $k e_{r}^{(r)}=0$. Then $\gamma:=\left\|\Pi_{C_{k} / / P(A, b, c ; 0)}\right\|_{\mathcal{W}_{0}, \mathcal{W}_{0}}<\infty$. Suppose $h \in\left(0, h^{*}\right)$, where $h^{*}>0$ satisfies (3.3). Then the delay feedback controller $C_{k}^{\text {Euler }}[h]: \mathcal{Y}_{0 e} \rightarrow \mathcal{U}_{0 e}$, defined in (1.2), applied to the plant $P(A, b, c ; 0): \mathcal{U}_{0 e} \rightarrow \mathcal{Y}_{0 e}$ given by (4.1) yields

$$
\left\|\Pi_{C_{k}^{\text {Euler }[h] / / P(A, b, c ; 0)}}\right\|_{\mathcal{W}_{0}, \mathcal{W}_{0}} \leq \gamma \frac{1+h \sum_{i=1}^{r-1}\left|k_{i+1}\right| i \eta_{p}(h, i)}{1-h \gamma \sum_{i=1}^{r-1}\left|k_{i+1}\right| i \eta_{p}(h, i)} .
$$

Proof. By Theorem 4.4 it follows that $\gamma:=\left\|\Pi_{C_{k} / / P(A, b, c ; 0)}\right\|_{\mathcal{W}_{0}, \mathcal{W}_{0}}<\infty$. The result now follows from Theorem 3.1 since $\left[P(A, b, c ; 0), C_{k}^{\text {Euler }}[h]\right]$ is globally well posed and $P(0)=0$.

In the following let $C_{k, \kappa, \nu}^{\text {Euler }}[h]: y_{2} \mapsto u_{2}=-\nu \sum_{i=0}^{r-1} \kappa^{r-i} k_{i+1} \Delta_{h}^{i} y_{2}$ be the delay feedback controller corresponding to controller $C_{k, \kappa, \nu}$ given in (4.2).

Together with Proposition 4.1 and Theorem 4.4, Theorem 4.5 shows for signal spaces of type (A) or (C) in (3.2), that for sufficiently large $\kappa, \nu \geq 1$ (determined by Proposition 4.1), $\beta(k, \kappa, \nu)$ given in (4.12), and sufficiently small $h>0$ (determined by Theorem 4.5), the closed-loop system $\left[P(A, b, c ; 0), C_{k, \kappa, \nu}^{\text {Euler }}[h]\right]$ is $\mathcal{W}_{0}$-gain stable and

$$
\left\|\Pi_{C_{k, \kappa, \nu}^{\text {Euler }}[h] / / P(A, b, c ; 0)}\right\|_{\mathcal{W}_{0}, \mathcal{W}_{0}} \leq \beta(k, \kappa, \nu) \frac{1+h \nu \kappa^{r-1} \sum_{i=1}^{r-1}\left|k_{i+1}\right| i \eta_{p}(h, i)}{1-h \beta(k, \kappa, \nu) \nu \kappa^{r-1} \sum_{i=1}^{r-1}\left|k_{i+1}\right| i \eta_{p}(h, i)} .
$$

\subsection{Gain stability of $\left[P\left(A, b, c ; x^{0}\right), C_{k}^{\text {Euler }}[h]\right]$ with non-zero initial condition}

To generalize Theorem 4.5 by allowing for non-zero initial conditions, we give the following result which will be applied to signal spaces of type (A) or (B) in (3.2). The proof of Theorem 4.6 is based on an extension of $[4$, Th. 5.3].

Theorem 4.6 Let $r, n \in \mathbb{N}$ with $r \leq n$ and consider signal spaces $\mathcal{U}, \mathcal{Y}, \mathcal{W}, \mathcal{W}_{0}$ of type (A) or (B) in (3.2). Let $k \in \mathbb{R}^{1 \times r}$ and additionally in case (B) suppose $k e_{r}^{(r)}=0$. Let $(A, b, c) \in \mathcal{P}_{n, r}$, $x^{0} \in \mathbb{R}^{n}$ and consider the operator $P\left(A, b, c ; x^{0}\right): \mathcal{U}_{e} \rightarrow \mathcal{Y}_{e}$ as defined in (4.1). Suppose that for $h>0$, applying the feedback controllers

$$
C_{k}: \mathcal{Y}_{e} \rightarrow \mathcal{U}_{e} \quad \text { and } \quad C_{k}^{\text {Euler }}[h]: \mathcal{Y}_{0 e} \rightarrow \mathcal{U}_{e}
$$

as defined in (1.1) and (1.2), respectively, to $P(A, b, c ; 0)$ yields

$$
\left\|\Pi_{C_{k} / / P(A, b, c ; 0)}\right\|_{\mathcal{W}_{0}, \mathcal{W}_{0}}<\infty \quad \text { and } \quad\left\|\Pi_{C_{k}^{\text {Euler }}[h] / / P(A, b, c ; 0)}\right\|_{\mathcal{W}_{0}, \mathcal{W}_{0}}=: \gamma<\infty
$$

Then

$$
\exists \lambda>0 \forall x^{0} \in \mathbb{R}^{n} \forall w_{0} \in \mathcal{W}_{0}:\left\|\Pi_{C_{k}^{\text {Euler }}[h] / / P\left(A, b, c ; x^{0}\right)} w_{0}\right\|_{\mathcal{W}} \leq \lambda\left|x^{0}\right|+\gamma\left\|w_{0}\right\|_{\mathcal{W}_{0}} .
$$

Proof. Note that we may consider $P(A, b, c ; 0)$ as an operator from $\mathcal{U}_{e}$ to $\mathcal{Y}_{e}$ or from $\mathcal{U}_{e}$ to $\mathcal{Y}_{0 e}$. Furthermore, note that $C_{k}$ and $C_{k}^{\text {Euler }}[h]$ may be considered as operators from $\mathcal{Y}_{e}$ to $\mathcal{U}$ or from $\mathcal{Y}_{0 e}$ to $\mathcal{U}$. Thus we may consider the graphs of $P(A, b, c ; 0), C_{k}$ and $C_{k}^{\text {Euler }}[h]$ in $\mathcal{W}_{0}$ or in $\mathcal{W}$. To identify in which signal space a graph is considered we add a superscript $\mathcal{W}_{0}$ or $\mathcal{W}$ such 
as in $\mathcal{G}_{P(A, b, c ; 0)}^{\mathcal{W}_{0}} \subset \mathcal{W}_{0}$ or $\mathcal{G}_{P(A, b, c ; 0)}^{\mathcal{W}} \subset \mathcal{W}$. For $x^{0} \neq 0$ we have to consider $P\left(A, b, c ; x^{0}\right)$ as an operator from $\mathcal{U}_{e}$ to $\mathcal{Y}_{e}$ with $\mathcal{G}_{P\left(A, b, c ; x^{0}\right)}^{\mathcal{W}} \subset \mathcal{W}$.

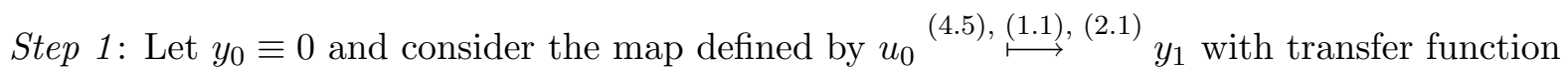

$$
\begin{aligned}
s \mapsto G(s) & =(1,0, \ldots, 0)\left(s I-\left(V A V^{-1}+V b(k \mid 0)\right)\right)^{-1} V b \\
& =(1,0, \ldots, 0)\left(V\left(s I-\left(A+b k\left[c / \ldots / c A^{r-1}\right]\right)\right) V^{-1}\right)^{-1} V b,
\end{aligned}
$$

where the matrix $V$ is given by (4.3). By boundedness of $\left\|H_{P(A, b, c ; 0), C_{k}}\right\|_{\mathcal{W}_{0}, \mathcal{W}_{0} \times \mathcal{W}_{0}}$ and $[3$, Th. 2, Sect. 2.4] it follows that $G(\cdot)$ is stable. Since $(A, b, c)$ is minimum phase, setting $F:=$ $k\left[c / \ldots / c A^{r-1}\right],\left[1\right.$, Th. 10] yields that $\operatorname{spec}(A+b F)=\operatorname{spec}\left(\left(A+b k\left[c / \ldots / c A^{r-1}\right]\right) \subset \mathbb{C}_{-}\right.$.

Since $\left\|H_{P(A, b, c ; 0), C_{k}}\right\|_{\mathcal{W}_{0}, \mathcal{W}_{0} \times \mathcal{W}_{0}}<\infty$, we may define maps $\tilde{N}: \mathcal{U} \rightarrow \mathcal{U}, u_{0} \mapsto u_{1}$, and $M: \mathcal{U} \rightarrow$ $\mathcal{Y}_{0}, u_{0} \mapsto y_{1}$ by

$$
\widetilde{N} u_{0}=\left(\begin{array}{ll}
1 & 0
\end{array}\right) \Pi_{P(A, b, c ; 0) / / C_{k}}\left(\begin{array}{c}
u_{0} \\
0
\end{array}\right), \quad M u_{0}=\left(\begin{array}{ll}
0 & 1
\end{array}\right) \Pi_{P(A, b, c ; 0) / / C_{k}}\left(\begin{array}{c}
u_{0} \\
0
\end{array}\right) .
$$

Proposition 4.1 yields that the tuples $\left(u_{0}, u_{1}\right)=\left(u_{0}, \widetilde{N} u_{0}\right)$ and $\left(u_{0}, y_{1}\right)=\left(u_{0}, M u_{0}\right)$ satisfy

$$
\begin{aligned}
\dot{x} & =(A+b F) x+b u_{0}, \quad x(0)=0 \\
u_{1} & =F x+u_{0} \\
y_{1} & =c x
\end{aligned}
$$

Step 2: We show $\widetilde{N}(\mathcal{U})=\mathcal{V}:=\{u \in \mathcal{U} \mid P(A, b, c ; 0) u \in \mathcal{Y}\}$.

Suppose $u \in \mathcal{V}$, i.e. $u \in \mathcal{U}$ with $P(A, b, c ; 0) u \in \mathcal{Y}$. Then $\mathcal{Y} \ni P(A, b, c ; 0) u=c x=: y$ for $x$ being a solution of $\dot{x}=A x+b u, x(0)=0$. Since $(A, b, c)$ is minimum phase, thus $(A, c)$ is detectable, there exists $L \in \mathbb{R}^{n}$ such that $\operatorname{spec}(A+L c) \subset \mathbb{C}_{-}$. Since $y \in \mathcal{Y}$ and $u \in \mathcal{U}$ writing

$$
\dot{x}=(A+L c) x-L c x+b u=(A+L c) x-L y+b u
$$

yields that $x \in C L^{p}\left(\mathbb{R}_{\geq 0} \rightarrow \mathbb{R}^{n}\right)$. Thus $u_{0}:=u-F x \in \mathcal{U}$ and (4.15) then yields that $u=u_{1}=$ $\widetilde{N}\left(u_{0}\right) \in \widetilde{N}(\mathcal{U})$, which gives $\tilde{N}(\mathcal{U}) \subset \mathcal{V}$.

Conversely, suppose $u \in N(\mathcal{U})$. Then there exists $u_{0} \in \mathcal{U}$ such that $u_{0}=u-F x \in \mathcal{U}$. Since $\operatorname{spec}(A+b F) \subset \mathbb{C}_{-}$it follows by (4.15) that $P(A, b, c ; 0) u=y=c x \in \mathcal{Y}$. Hence $\widetilde{N}(\mathcal{U}) \subset \mathcal{V}$.

Now $N: \mathcal{U} \rightarrow \mathcal{V}, u_{0} \mapsto\left(\begin{array}{ll}1 & 0\end{array}\right) \Pi_{P(A, b, c ; 0) / / C_{k}}\left(\begin{array}{c}u_{0} \\ 0\end{array}\right)$, is well defined and writing

$$
\begin{aligned}
\dot{x} & =A x+b u_{1}, \quad x(0)=0 \\
u_{0} & =F x-u_{1},
\end{aligned}
$$

directly gives that is invertible and $P(A, b, c ; 0)=M N^{-1}$.

Step 3: Set $\bar{A}:=A+b F=\left(A+b k\left[c / \ldots / c A^{r-1}\right]\right)$. We show

$$
\left.\mathcal{G}_{P\left(A, b, c ; x^{0}\right)}^{\mathcal{W}}=Q:=\left\{\begin{array}{l}
N \\
M
\end{array}\right) v+\left(\begin{array}{l|l}
F \exp (\bar{A} \cdot) x^{0} \\
c \exp (\bar{A} \cdot) x^{0}
\end{array}\right) \in \mathcal{W} \mid \begin{array}{l}
v \in \mathcal{U}, \\
N, M, F \text { and } \bar{A} \text { as in Step 1 }
\end{array}\right\} .
$$

We show $Q \subset \mathcal{G}_{P\left(A, b, c ; x^{0}\right)}^{\mathcal{W}}$. Consider, for any $v \in \mathcal{U}, q_{v}=\left(\begin{array}{c}N \\ M\end{array}\right) v+\left(\begin{array}{c}F \exp (\bar{A} \cdot) x^{0} \\ c \exp (\bar{A} \cdot) x^{0}\end{array}\right) \in Q$. Let $u=N v+F \exp (\bar{A} \cdot) x^{0}$. Since $N v \in \mathcal{U}$ and $\exp (\bar{A} \cdot) \in C W^{r, p}\left(\mathbb{R}_{\geq 0} \rightarrow \mathbb{R}\right)^{n \times n}=\mathcal{Y}^{n \times n}$, we have $u \in \mathcal{U}$. Observe

$$
\dot{x}=A x+b\left(F \exp (\bar{A} \cdot) x^{0}\right), \quad x(0)=x^{0} \in \mathbb{R}^{n}
$$


has the solution $x(\cdot)=\exp (\bar{A} \cdot) x^{0}$. Thus it follows that

$$
P\left(A, b, c ; x^{0}\right) F \exp (\bar{A} \cdot) x^{0}=c \exp (\bar{A} \cdot) x^{0} .
$$

Hence

$$
\begin{aligned}
P\left(A, b, c ; x^{0}\right) u & =P\left(A, b, c ; x^{0}\right) N v+P\left(A, b, c ; x^{0}\right)\left(F \exp (\bar{A} \cdot) x^{0}\right)-P\left(A, b, c ; x^{0}\right) 0 \\
& =P(A, b, c ; 0) N v+P\left(A, b, c ; x^{0}\right)\left(F \exp (\bar{A} \cdot) x^{0}\right) \\
& =M(N)^{-1} N v+c \exp (\bar{A} \cdot) x^{0} \\
& =M v+c \exp (\bar{A} \cdot) x^{0} \in \mathcal{Y} .
\end{aligned}
$$

Thus $q_{v}=\left(\begin{array}{c}u \\ P\left(A, b, c ; x^{0}\right) u\end{array}\right) \in \mathcal{U} \times \mathcal{Y}$, so $q_{v} \in \mathcal{G}_{P\left(A, b, c ; x^{0}\right)}^{\mathcal{W}}$ and $Q \subset \mathcal{G}_{P\left(A, b, c ; x^{0}\right)}^{\mathcal{W}}$.

We show $\mathcal{G}_{P\left(A, b, c ; x^{0}\right)}^{\mathcal{W}} \subset Q$. Consider $\left(\begin{array}{c}u \\ P\left(A, b, c ; x^{0}\right) u\end{array}\right) \in \mathcal{G}_{P\left(A, b, c ; x^{0}\right)}^{\mathcal{W}}$. Then

$$
P(A, b, c ; 0)\left(u-F \exp (\bar{A} \cdot) x^{0}\right)=P\left(A, b, c ; x^{0}\right) u-P\left(A, b, c ; x^{0}\right)\left(F \exp (\bar{A} \cdot) x^{0}\right)
$$

and since the right hand side lies in $\mathcal{Y}$, it follows that $P(A, b, c ; 0)\left(u-F \exp (\bar{A} \cdot) x^{0}\right) \in \mathcal{Y}$. Therefore $u-F \exp (\bar{A} \cdot) x^{0} \in \mathcal{V}=\operatorname{Im}(N)$, and so there exists $v \in \mathcal{U}$ such that $N v=u-$ $F \exp (\bar{A} \cdot) x^{0}$. Therefore equation (4.16) holds, hence

$$
\left(\begin{array}{c}
u \\
P\left(A, b, c ; x^{0}\right) u
\end{array}\right)=\left(\begin{array}{c}
N \\
M
\end{array}\right) v+\left(\begin{array}{c}
F \exp (\bar{A} \cdot) x^{0} \\
c \exp (\bar{A} \cdot) x^{0}
\end{array}\right) \in Q
$$

and so $\mathcal{G}_{P\left(A, b, c ; x^{0}\right)}^{\mathcal{W}} \subset Q$. Therefore we have shown $\mathcal{G}_{P\left(A, b, c ; x^{0}\right)}^{\mathcal{W}}=Q$ as claimed.

Step 4: Finally we show (4.14). For $w_{0} \in \mathcal{W}_{0}$ and $x^{0} \in \mathbb{R}^{n}$ let

$$
w_{0}^{\prime}:=w_{0}-v_{1}-v_{2}, \quad v_{1}:=\left(\begin{array}{c}
F \exp (\bar{A} \cdot) x^{0} \\
c \exp (\bar{A} \cdot) x^{0}
\end{array}\right) \quad v_{2}:=\left(\begin{array}{c}
-C_{k}^{\text {Euler }}[h]\left(c \exp (\bar{A} \cdot) x^{0}\right) \\
-c \exp (\bar{A} \cdot) x^{0}
\end{array}\right) .
$$

Since $C_{k}^{\text {Euler }}[h](\mathcal{Y}) \subset \mathcal{U}$, we have $w_{0}^{\prime} \in \mathcal{W}_{0}$, hence,

$$
H_{P(A, b, c ; 0), C_{k}^{\text {Euler }[h]}}\left(w_{0}^{\prime}\right)=\left(w_{1}, w_{2}\right) \in \mathcal{G}_{P(A, b, c ; 0)}^{\mathcal{W}_{0}} \times \mathcal{G}_{C_{k}^{\text {Euler }}[h]}^{\mathcal{W}_{0}} .
$$

In particular, $w_{0}^{\prime}=w_{1}+w_{2}$, and by rearranging we have $w_{0}=\left(w_{1}+v_{1}\right)+\left(w_{2}+v_{2}\right)$. Since $w_{1} \in \mathcal{G}_{P(A, b, c ; 0)}^{\mathcal{W}_{0}} \subset \mathcal{G}_{P(A, b, c ; 0)}^{\mathcal{W}}$, there exists $v \in \mathcal{U}$ such that $w_{1}=\left(\begin{array}{c}N \\ M\end{array}\right) v$, hence $w_{1}+v_{1} \in Q=\mathcal{G}_{P\left(A, b, c ; x^{0}\right)}^{\mathcal{W}}$. Since $w_{2} \in \mathcal{G}_{C_{k}^{\text {Euler }}[h]}^{\mathcal{W}_{0}} \subset \mathcal{G}_{C_{k}^{\text {Euler }}[h]}^{\mathcal{W}}$ and $v_{2} \in \mathcal{G}_{C_{k}^{\text {Euler }}[h]}^{\mathcal{W}}$, it follows by linearity of $C_{k}^{\text {Euler }}[h]$ that $w_{2}+v_{2} \in \mathcal{G}_{C_{k}^{\text {Euler }}[h]}^{\mathcal{W}}$. Therefore, since $\left[P\left(A, b, c ; x^{0}\right), C_{k}^{\text {Euler }}[h]\right]$ has the uniqueness property, $H_{P\left(A, b, c ; x^{0}\right), C_{k}^{\text {Euler }}[h]}: \mathcal{W} \rightarrow \mathcal{W} \times \mathcal{W}$ is defined and

$$
H_{P\left(A, b, c ; x^{0}\right), C_{k}^{\text {Euler }[h]}} w_{0}=\left(w_{1}+v_{1}, w_{2}+v_{2}\right) \in \mathcal{G}_{P\left(A, b, c ; x^{0}\right)}^{\mathcal{W}} \times \mathcal{G}_{C_{k}^{\text {Euler }[h]}}^{\mathcal{W}} \subset \mathcal{W} \times \mathcal{W} .
$$

Now for

$$
\lambda:=\sup _{x_{0} \in \mathbb{R}^{n} \backslash\{0\}} \frac{\left\|v_{2}\right\|_{\mathcal{W}}}{\left|x^{0}\right|}=\left\|\left(\begin{array}{c}
-C_{k}^{\text {Euler }}[h](c \exp (\bar{A} \cdot)) \\
-c \exp (\bar{A} \cdot)
\end{array}\right)\right\|_{\mathcal{W}^{n}}
$$

it follows that

$$
\left\|\Pi_{C_{k}^{\text {Euler }}[h] / / P\left(A, b, c ; x^{0}\right)} w_{0}\right\|_{\mathcal{W} \times \mathcal{W}} \leq\left\|v_{2}\right\|_{\mathcal{W}}+\left\|w_{2}\right\|_{\mathcal{W}} \leq \lambda\left|x^{0}\right|+\gamma\left\|w_{0}\right\|_{\mathcal{W}_{0}},
$$

thus concluding the proof. 
We can now state the result for the delay feedback controller in the presence of both input/ouput disturbances and initial conditions.

Theorem 4.7 Let, for $r, n \in \mathbb{N}$ with $r \leq n,(A, b, c) \in \mathcal{P}_{n, r}$ and choose $k \in \mathbb{R}^{1 \times r}$ such that $\operatorname{spec}\left(A+b k\left[c / \ldots / c A^{r-1}\right]\right) \subset \mathbb{C}_{-}$. Let the signal spaces $\mathcal{U}, \mathcal{Y}, \mathcal{W}, \mathcal{W}_{0}$ be of type (A) or (B) in (3.2); in case of (B) suppose $k e_{r}^{(r)}=0$. Then $\gamma:=\left\|\Pi_{C_{k} / / P(A, b, c ; 0)}\right\|_{\mathcal{W}_{0}, \mathcal{W}_{0}}<\infty$. Suppose $h \in\left(0, h^{*}\right)$, where $h^{*}>0$ satisfies (3.3). Consider for $(A, b, c)$ the plant operator $P\left(A, b, c ; x^{0}\right): \mathcal{U}_{e} \rightarrow \mathcal{Y}_{e}$ given by $(4.1)$ and the delay feedback controller $C_{k}^{\text {Euler }}[h]: \mathcal{Y}_{0 e} \rightarrow \mathcal{U}_{e}$ defined in (1.2). Then there exists $\lambda>0$ such that, for all $w_{0} \in \mathcal{W}_{0}$,

$$
\left\|\Pi_{C_{k}^{\text {Euler }}[h] / / P\left(A, b, c ; x^{0}\right)} w_{0}\right\|_{\mathcal{W}, \mathcal{W}} \leq \lambda\left|x^{0}\right|+\gamma \frac{1+h \sum_{i=1}^{r-1}\left|k_{i+1}\right| i \eta_{p}(h, i)}{1-h \gamma \sum_{i=1}^{r-1}\left|k_{i+1}\right| i \eta_{p}(h, i)}\left\|w_{0}\right\|_{\mathcal{W}_{0}} .
$$

Proof. The result follows directly from Theorem 4.5 and Theorem 4.6.

Together with Proposition 4.1 and Theorem 4.4, Theorem 4.7 shows for signal spaces of type (A) in (3.2), that for sufficiently large $\kappa, \nu \geq 1$ (determined by Proposition 4.1), $\beta(k, \kappa, \nu)$ given in (4.12) and for sufficiently small $h>0$ (determined by Theorem 4.5) there exists $\lambda>0$ (determined by equation (4.17)) such that, for all $x_{0} \in \mathbb{R}^{n}$ and $w_{0} \in \mathcal{W}_{0}$ :

$$
\begin{aligned}
&\left\|\Pi_{C_{k, \kappa, \nu}^{\text {Euler }}[h] / / P\left(A, b, c ; x^{0}\right)} w_{0}\right\|_{\mathcal{W}, \mathcal{W}} \\
& \leq \lambda\left|x^{0}\right|+\left(\beta(k, \kappa, \nu) \frac{1+h \nu \kappa^{r-1} \sum_{i=1}^{r-1}\left|k_{i+1}\right| i \eta_{p}(h, i)}{1-h \beta(k, \kappa, \nu) \nu \kappa^{r-1} \sum_{i=1}^{r-1}\left|k_{i+1}\right| i \eta_{p}(h, i)}\right)\left\|w_{0}\right\|_{\mathcal{W}_{0}} .
\end{aligned}
$$

\subsection{Exponential stability of $\left[P\left(A, b, c ; x^{0}\right), C_{k}^{\text {Euler }}[h]\right]$ with $u_{0} \equiv y_{0} \equiv 0$}

In Proposition 4.1 we have shown that the high-gain derivative feedback controller $C_{k, \kappa, \nu}: y_{2} \mapsto$ $u_{2}$ leads to an internally stable system, i.e. (4.7) with $u_{0} \equiv y_{0} \equiv 0$ gives

$$
\exists \nu^{*} \geq 1 \forall \nu \geq \nu^{*} \exists \kappa^{*} \geq 1, \forall \kappa \geq \kappa^{*} \quad: \dot{z}=A_{k, \kappa, \nu} z \quad \text { is exponentially stable. }
$$

Now (as in [10] where a more limited class of systems was considered) we will show that an analogous result holds true if a stabilizing derivative feedback controller $C_{k}: y_{2} \mapsto u_{2}$ is replaced by the delay feedback $C_{k}^{\text {Euler }}[h]: y_{2} \mapsto u_{2}$ for $h>0$ sufficiently small. Exponential stability for a delay differential equation is defined as follows, see, for example [2, Def. 5.1.1].

Definition 4.8 Let $h>0$ and, for $r, n \in \mathbb{N}$ with $r \leq n, A_{0}, \ldots, A_{r-1} \in \mathbb{R}^{n \times n}$. Then the delay initial value problem

$$
\dot{x}=\sum_{j=0}^{r-1} A_{j} x(t-j h), \quad x \equiv \varphi \text { on }[(1-r) h, 0],
$$

is said to be exponentially stable if, and only if,

$$
\exists M, \lambda>0 \forall t \geq 0 \forall \varphi \in \mathcal{C}_{\mathrm{pw}}\left([(1-r) h, 0] \rightarrow \mathbb{R}^{n}\right):|x(t)| \leq M e^{-\lambda t} \max _{s \in[(1-r) h, 0]}|\varphi(s)| .
$$


Proposition 4.9 Let, for $r, n \in \mathbb{N}$ with $r \leq n,(A, b, c) \in \mathcal{P}_{n, r}, x^{0} \in \mathbb{R}^{n}$. Consider the signal spaces $\mathcal{U}=\mathcal{Y}=C W_{0}^{\infty, 2}\left(\mathbb{R}_{\geq 0} \rightarrow \mathbb{R}\right)$ and $\mathcal{W}:=\mathcal{U} \times \mathcal{Y}$ and choose $k \in \mathbb{R}^{1 \times r}$ and $h>0$ such that

$$
\left\|\Pi_{P(A, b, c ; 0) / / C_{k}^{\text {Euler }[h]}}\right\|_{\mathcal{W}, \mathcal{W}}<\infty
$$

Then the delay initial value problem for the closed-loop system $\left[P\left(A, b, c ; x^{0}\right), C_{k}^{\text {Euler }}[h]\right]$ given by (4.1), (1.2) and (2.1) with $u_{0} \equiv y_{0} \equiv 0$ is exponentially stable.

Proof. For $(A, b, c) \in \mathcal{P}_{n, r}, r, n \in \mathbb{N}$ with $r \leq n$ and $h>0$, the closed-loop system $\left[P\left(A, b, c ; x^{0}\right), C_{k}^{\text {Euler }}[h]\right]$ given by $(4.1),(1.2)$ and $(2.1)$ is described by a delay differential equation of the form (4.18) as follows:

$$
\left.\begin{array}{rl}
\dot{x}(t) & =\left(A+\widetilde{A}^{0}\right) x(t)+\sum_{j=1}^{r-1} \widetilde{A}^{j} x(t-j h)+b u_{0}(t)+\sum_{j=0}^{r-1} \widetilde{b}^{j} y_{0}(t-j h), \\
y_{1}(t) & =c x(t), \\
u_{1}(t) & =u_{0}(t)+\sum_{j=0}^{r-1}(-1)^{j} \sum_{i=j}^{r-1} \frac{k_{i+1}}{h^{i}}\left(\begin{array}{l}
i \\
j
\end{array}\right)\left(y_{0}-y_{1}\right)(t-j h)
\end{array}\right\}
$$

where $\varphi(0)=x^{0}$ and, in view of $(4.4),(4.5)$, for $j=0, \ldots, r-1$,

$$
\widetilde{A}^{j}:=(-1)^{j+1} c A^{r-1} b \sum_{i=j}^{r-1} \frac{k_{i+1}}{h^{i}}\left(\begin{array}{l}
i \\
j
\end{array}\right) V^{-1}\left[e_{r}^{(n)} \mid 0_{n \times(n-1)}\right] V, \quad \widetilde{b}^{j}:=(-1)^{j} \sum_{i=j}^{r-1} \frac{k_{i+1}}{h^{i}}\left(\begin{array}{l}
i \\
j
\end{array}\right) b,
$$

and the transformation matrix $V \in \mathbb{R}^{n \times n}$ is given by (4.3). Let $G_{P(A, b, c ; 0) / / C_{k}^{\text {Euler }[h]}} \in \mathbb{R}(s)^{2 \times 2}$ denote the transfer function of $\Pi_{P(A, b, c ; 0) / / C_{k}^{\text {Euler }}[h]}$. Then, [16, Th. 30] and [3, Th. 2, Sect. 2.4] yields:

$$
\sup _{\omega \in \mathbb{R}}\left\|G_{P(A, b, c ; 0) / / C_{k}^{\text {Euler }[h]}}(i \omega)\right\|_{2}=\left\|\Pi_{P(A, b, c ; 0) / / C_{k}^{\text {Euler }[h]}}\right\|_{\mathcal{W}, \mathcal{W}}<\infty .
$$

Since the denominator of the function $\left(s \mapsto G_{P(A, b, c ; 0) / / C_{k}^{\text {Euler }}[h]}(s)\right)$ is equal to

$$
\operatorname{det}\left(s I-\left(A+\widetilde{A}^{0}+e^{-s h} \widetilde{A}^{1}+\cdots+e^{-s(r-1) h} \widetilde{A}^{r-1}\right)\right),
$$

it follows that

$$
\forall s \in \overline{\mathbb{C}}_{+}: \operatorname{det}\left(s I-\left(A+\widetilde{A}^{0}+e^{-s h} \widetilde{A}^{1}+\cdots+e^{-s(r-1) h} \widetilde{A}^{r-1}\right)\right) \neq 0 .
$$

Now, [2, Th. 5.1.5] yields exponential stability of (4.20) with $u_{0} \equiv y_{0} \equiv 0$.

We conclude the paper by noting that for sufficiently large $\kappa, \nu \geq 1$ (determined by Proposition 4.1) and for sufficiently small $h>0$ (determined by Theorem 4.5) Proposition 4.9 yields that the closed-loop system $\left[P\left(A, b, c ; x^{0}\right), C_{k, \kappa, \nu}^{\text {Euler }}[h]\right]$ with $u_{0} \equiv y_{0} \equiv 0$ is exponentially stable.

\section{References}

[1] W. Coppel. Matrices of rational functions. Bull. Austr. Math. Soc., 11:89-113, 1974.

[2] R. F. Curtain and H. J. Zwart. An introduction to infinite-dimensional linear systems theory. Number 21 in Texts in Applied Mathematics. Springer-Verlag, New York, 1995. 
[3] B. A. Francis. A course in $H_{\infty}$ control theory. Number 88 in Lecture Notes in Control and Information Sciences. Springer-Verlag, Berlin-Heidelberg-New York, 1987.

[4] M. French. Adaptive control and robustness in the gap metric. IEEE Trans. Autom. Control, 53(2):461-478, 2008.

[5] M. French, A. Ilchmann, and E. Ryan. Robustness in the graph topology of a common adaptive controller. SIAM J. Control Optim., 45(5):1736-1757, 2006.

[6] M. French and M. Mueller. Nonlinear high-gain separation principles and fast sampling results ensuring robust stability. in preperation.

[7] T. Georgiou and M. Smith. Robustness analysis of nonlinear feedback systems: An inputoutput approach. IEEE Trans. Autom. Control, 42(9):1200-1221, 1997.

[8] T. T. Georgiou and M. C. Smith. Graphs, causality, and stabilizability: Linear, shiftinvariant systems on $\mathfrak{L}_{2}[0, \infty)$. Math. Control Signals Syst., 6(3):195-223, 1992.

[9] A. Ilchmann, E. P. Ryan, and P. Townsend. Tracking with prescribed transient behavior for nonlinear systems of known relative degree. SIAM J. Control Optim., 46(1):210-230, 2007.

[10] A. Ilchmann and C. Sangwin. Output feedback stabilisation of minimum phase systems by delays. Syst. Control Lett., 52(3):233-245, 2004.

[11] I. Karafyllis. Finite-time global stabilization by means of time-varying distributed delay feedback. SIAM J. Control Optim., 45(1):320-342, 2006.

[12] V. Kharitonov, S.-J. Niculescu, J. Moreno, and W. Michiels. Static output feedback stabilisation: Necessary conditions for multiple delay controllers. IEEE Trans. Autom. Control, $50(1): 82-86,2005$.

[13] S.-I. Niculescu and W. Michiels. Stabilizing a chain of integrators using multiple delays. IEEE Trans. Autom. Control, 49(5):802-807, 2004.

[14] K. Ogata. Modern Control Engineering. Prentice-Hall, Englewood Cliffs, NJ, 2nd edition, 1990.

[15] T. Rivlin. An introduction to the approximation of functions. Blaisdell Publishing Company, Waltham, Mass., 1969.

[16] S. Trenn. Hardy spaces and robustness of linear systems. Diploma Thesis, Ilmenau Technical University, Ilmenau, 2004, http://tu-ilmenau.de/fakmn/Publications.3187.0.html

[17] K. Yosida. Functional analysis. Springer-Verlag, Berlin, 6th edition, 1980. 